\title{
DIFFERENTIAL EQUATIONS FOR SYMMETRIC GENERALIZED ULTRASPHERICAL POLYNOMIALS
}

\author{
ROELOF KOEKOEK
}

ABSTRACT. We look for differential equations satisfied by the generalized Jacobi polynomials $\left\{P_{n}^{\alpha, \beta, M, N}(x)\right\}_{n=0}^{\infty}$ which are orthogonal on the interval $[-1,1]$ with respect to the weight function

$$
\frac{\Gamma(\alpha+\beta+2)}{2^{\alpha+\beta+1} \Gamma(\alpha+1) \Gamma(\beta+1)}(1-x)^{\alpha}(1+x)^{\beta}+M \delta(x+1)+N \delta(x-1),
$$

where $\alpha>-1, \beta>-1, M \geq 0$, and $N \geq 0$.

In the special case that $\beta=\alpha$ and $N=M$ we find all differential equations of the form

$$
\sum_{i=0}^{\infty} c_{i}(x) y^{(i)}(x)=0, \quad y(x)=P_{n}^{\alpha, \alpha, M, M}(x),
$$

where the coefficients $\left\{c_{i}(x)\right\}_{i=1}^{\infty}$ are independent of the degree $n$.

We show that if $M>0$ only for nonnegative integer values of $\alpha$ there exists exactly one differential equation which is of finite order $2 \alpha+4$.

By using quadratic transformations we also obtain differential equations for the polynomials $\left\{P_{n}^{\alpha, \pm 1 / 2,0, N}(x)\right\}_{n=0}^{\infty}$ for all $\alpha>-1$ and $N \geq 0$.

\section{INTRODUCTION}

In the late thirties (see [10] and [11]) H. L. Krall classified all sets of orthogonal polynomials $\left\{P_{n}(x)\right\}_{n=0}^{\infty}$ with degree $\left[P_{n}(x)\right]=n$ which satisfy a fourthorder differential equation of the form

$$
p_{4}(x) y^{(4)}(x)+p_{3}(x) y^{(3)}(x)+p_{2}(x) y^{\prime \prime}(x)+p_{1}(x) y^{\prime}(x)+p_{0}(x) y(x)=0
$$

where $\left\{p_{i}(x)\right\}_{i=0}^{4}$ are polynomials with degree $\left[p_{i}(x)\right] \leq i$ and $\left\{p_{i}(x)\right\}_{i=1}^{4}$ are independent of the degree $n$. These sets of orthogonal polynomials include the classical Legendre, Laguerre, Hermite, Bessel, and Jacobi polynomials. He also found three other sets of orthogonal polynomials satisfying a fourth-order differential equation of this type. In [8] A. M. Krall studied these new sets of orthogonal polynomials in more detail and named them the Legendre type, Laguerre type, and Jacobi type polynomials. These polynomials are generalizations of the classical Legendre, Laguerre (with $\alpha=0$ ), and Jacobi polynomials (with $\beta=0$ ) in the sense that the weight function for these orthogonal polynomials consists of the classical weight function together with a Dirac delta function at the end point(s) of the interval of orthogonality.

Received by the editors April 28, 1992.

1991 Mathematics Subject Classification. Primary 33C45, 33A65; Secondary 34A35. 
Later L. L. Littlejohn (see [12]) studied a generalization of the Legendre type polynomials and named them after H. L. Krall: the Krall polynomials. These Krall polynomials are orthogonal on the interval $[-1,1]$ with respect to the weight function

$$
\frac{1}{A} \delta(x+1)+\frac{1}{B} \delta(x-1)+C, \quad A>0, B>0, \text { and } C>0 .
$$

In general $(A \neq B)$, these polynomials do not fit in the class of polynomials which satisfy a fourth-order differential equation of the above type. The Krall polynomials satisfy a sixth-order differential equation of a similar form.

A. M. Krall and L. L. Littlejohn did some work on the classification of higherorder differential equations having orthogonal polynomial solutions. They tried to classify all differential equations of the form

$$
\sum_{i=0}^{r} p_{i}(x) y^{(i)}(x)=0, \quad r \in\{2,3,4, \ldots\},
$$

where $\left\{p_{i}(x)\right\}_{i=0}^{r}$ are polynomials with degree $\left[p_{i}(x)\right] \leq i$ and $\left\{p_{i}(x)\right\}_{i=1}^{r}$ are independent of $n$ having orthogonal polynomial solutions $\left\{P_{n}(x)\right\}_{n=0}^{\infty}$ with degree $\left[P_{n}(x)\right]=n$. See [14] and [9].

In [7] T. H. Koornwinder found a general class of orthogonal polynomials which generalize the Legendre type, Jacobi type, and Krall polynomials. These polynomials are orthogonal on the interval $[-1,1]$ with respect to the weight function

$$
\frac{\Gamma(\alpha+\beta+2)}{2^{\alpha+\beta+1} \Gamma(\alpha+1) \Gamma(\beta+1)}(1-x)^{\alpha}(1+x)^{\beta}+M \delta(x+1)+N \delta(x-1),
$$

where $\alpha>-1, \beta>-1, M \geq 0$, and $N \geq 0$. For these generalized Jacobi polynomials we will use Koornwinder's notation: $\left\{P_{n}^{\alpha, \beta, M, N}(x)\right\}_{n=0}^{\infty}$.

As a limit case he found the polynomials $\left\{L_{n}^{\alpha}, M(x)\right\}_{n=0}^{\infty}$ which are orthogonal on the interval $[0, \infty)$ with respect to the weight function

$$
\frac{1}{\Gamma(\alpha+1)} x^{\alpha} e^{-x}+M \delta(x), \quad \alpha>-1 \text { and } M \geq 0 .
$$

These polynomials generalize the classical Laguerre polynomials.

In [5] J. Koekoek and R. Koekoek showed that the polynomials $\left\{L_{n}^{\alpha, M}(x)\right\}_{n=0}^{\infty}$ satisfy a unique differential equation of the form

$$
M \sum_{i=0}^{\infty} a_{i}(x) y^{(i)}(x)+x y^{\prime \prime}(x)+(\alpha+1-x) y^{\prime}(x)+n y(x)=0,
$$

where $\left\{a_{i}(x)\right\}_{i=0}^{\infty}$ are continuous functions on the real line and $\left\{a_{i}(x)\right\}_{i=1}^{\infty}$ are independent of $n$. It turns out that the coefficients $\left\{a_{i}(x)\right\}_{i=0}^{\infty}$ are polynonials and the differential equation is of infinite order in general if $M>0$. However, only for nonnegative integer values of $\alpha$ did the order reduce to $2 \alpha+4$.

We note that it is well known that all sets of polynomials named before satisfy a second-order differential equation with polynomial coefficients depending on $n$ but of bounded degree. See for instance [7], [13], and [15].

In this paper we look for differential equations satisfied by the polynomials $\left\{P_{n}^{\alpha, \beta, M, N}(x)\right\}_{n=0}^{\infty}$ with $\beta=\alpha$ and $N=M$. Until now, only two special cases, 
due to H. L. Krall and L. L. Littlejohn, are known. In [10] H. L. Krall showed that the polynomials $\left\{P_{n}^{0,0, M, M}(x)\right\}_{n=0}^{\infty}$ satisfy the following fourth-order (if $M>0$ ) differential equation:

$$
\begin{aligned}
& -\frac{1}{2} M\left(1-x^{2}\right)^{2} y^{(4)}(x)+4 M x\left(1-x^{2}\right) y^{(3)}(x) \\
& \quad+(6 M+1)\left(1-x^{2}\right) y^{\prime \prime}(x)-2 x y^{\prime}(x) \\
& \quad+\frac{1}{2} n(n+1)[(n-1)(n+2) M+2] y(x)=0
\end{aligned}
$$

and later L. L. Littlejohn found the following sixth-order (if $M>0$ ) differential equation for the polynomials $\left\{P_{n}^{1,1, M, M}(x)\right\}_{n=0}^{\infty}$ :

$$
\begin{aligned}
\frac{1}{36} M & \left(1-x^{2}\right)^{3} y^{(6)}(x)-\frac{2}{3} M x\left(1-x^{2}\right)^{2} y^{(5)}(x)-\frac{5}{3} M\left(1-x^{2}\right)\left(1-3 x^{2}\right) y^{(4)}(x) \\
& +\frac{40}{3} M x\left(1-x^{2}\right) y^{(3)}(x)+(10 M+1)\left(1-x^{2}\right) y^{\prime \prime}(x) \\
& -4 x y^{\prime}(x)+\frac{1}{36} n(n+3)[(n-1)(n+1)(n+2)(n+4) M+36] y(x)=0
\end{aligned}
$$

both in a different notation. The latter sixth-order differential equation has not appeared in the literature yet.

In this paper we will derive all differential equations for the polynomials $\left\{P_{n}^{\alpha, \alpha, M, M}(x)\right\}_{n=0}^{\infty}$ for every $\alpha>-1$ and $M \geq 0$, which are of the form

$$
\sum_{i=0}^{\infty} c_{i}(x) y^{(i)}(x)=0,
$$

where the coefficients $\left\{c_{i}(x)\right\}_{i=0}^{\infty}$ are continuous functions on the real line and $\left\{c_{i}(x)\right\}_{i=1}^{\infty}$ are independent of the degree $n$.

So we consider the polynomials $\left\{P_{n}^{\alpha, \alpha, M, M}(x)\right\}_{n=0}^{\infty}$ which can be defined by (see [7], in a slightly different notation)

$$
P_{n}^{\alpha, \alpha, M, M}(x)=C_{0} P_{n}^{(\alpha, \alpha)}(x)-C_{1} x \frac{d}{d x} P_{n}^{(\alpha, \alpha)}(x)
$$

where

$$
\left\{\begin{array}{l}
C_{0}=1+M \frac{2 n}{(\alpha+1)}\left(\begin{array}{c}
n+2 \alpha+1 \\
n
\end{array}\right)+4 M^{2}\left(\begin{array}{c}
n+2 \alpha+1 \\
n-1
\end{array}\right)^{2}, \\
C_{1}=\frac{2 M}{(2 \alpha+1)}\left(\begin{array}{c}
n+2 \alpha \\
n
\end{array}\right)+\frac{2 M^{2}}{(\alpha+1)}\left(\begin{array}{c}
n+2 \alpha \\
n-1
\end{array}\right)\left(\begin{array}{c}
n+2 \alpha+1 \\
n
\end{array}\right) .
\end{array}\right.
$$

As Koornwinder already remarked (see [7]) the case $2 \alpha+1=0$ must be understood by continuity in $\alpha$.

Further we will show that for $M>0$ these differential equations are of infinite order in general and only for nonnegative integer values of $\alpha$ we find exactly one differential equation of finite order $2 \alpha+4$. This answers one of the questions raised in [4] by W. N. Everitt and L. L. Littlejohn.

Finally, we will also derive differential equations for the polynomials $\left\{P_{n}^{\alpha, \pm 1 / 2,0, N}(x)\right\}_{n=0}^{\infty}$ for all $\alpha>-1$ and $N \geq 0$. 


\section{SOME ClASSICAL FORMULAS}

In this section we give the definition and some properties of the classical ultraspherical polynomials $\left\{P_{n}^{(\alpha, \alpha)}(x)\right\}_{n=0}^{\infty}$. For details the reader is referred to [2] and [16]. We will only give those properties we need in this paper. Further we list some other classical formulas which we will use later on.

The polynomials $\left\{P_{n}^{(\alpha, \alpha)}(x)\right\}_{n=0}^{\infty}$ can be defined by their representation as a hypergeometric function as

$$
\begin{aligned}
& P_{n}^{(\alpha, \alpha)}(x)=\left(\begin{array}{c|c}
n+\alpha \\
n
\end{array}\right){ }_{2} F_{1}\left(\begin{array}{c}
-n, n+2 \alpha+1 \\
\alpha+1
\end{array} \mid \frac{1-x}{2}\right), \\
& n=0,1,2, \ldots \text {. }
\end{aligned}
$$

A simple consequence of this definition is the differentiation formula

$$
\begin{aligned}
D^{i} P_{n}^{(\alpha, \alpha)}(x) & =\left(\begin{array}{c}
n+\alpha \\
n
\end{array}\right)\left(-\frac{1}{2}\right)^{i} \sum_{k=i}^{\infty} \frac{(-n)_{k}(n+2 \alpha+1)_{k}}{(k-i) !(\alpha+1)_{k}}\left(\frac{1-x}{2}\right)^{k-i} \\
& =\left(\begin{array}{c}
n+\alpha \\
n
\end{array}\right)\left(-\frac{1}{2}\right)^{i} \sum_{k=0}^{\infty} \frac{(-n)_{k+i}(n+2 \alpha+1)_{k+i}}{k !(\alpha+1)_{k+i}}\left(\frac{1-x}{2}\right)^{k}, \\
i & =0,1,2, \ldots
\end{aligned}
$$

Further we have the well-known symmetry relation

$$
P_{n}^{(\alpha, \alpha)}(-x)=(-1)^{n} P_{n}^{(\alpha, \alpha)}(x), \quad n=0,1,2, \ldots
$$

The ultraspherical polynomials satisfy a second-order linear differential equation given by

$$
\left(1-x^{2}\right) y^{\prime \prime}(x)-2(\alpha+1) x y^{\prime}(x)+n(n+2 \alpha+1) y(x)=0 .
$$

By using induction it is easy to show that this differential equation implies that

$$
\begin{aligned}
& \left(1-x^{2}\right) D^{i+2} P_{n}^{(\alpha, \alpha)}(x)-2(\alpha+i+1) x D^{i+1} P_{n}^{(\alpha, \alpha)}(x) \\
& \quad+(n-i)(n+2 \alpha+i+1) D^{i} P_{n}^{(\alpha, \alpha)}(x)=0, \quad i=0,1,2, \ldots
\end{aligned}
$$

We also need the following formulas for the even- and odd-order ultraspherical polynomials:

$$
\begin{array}{r}
P_{2 n}^{(\alpha, \alpha)}(x)=\left(-\frac{1}{4}\right)^{n}\left(\begin{array}{c}
2 n+\alpha \\
n
\end{array}\right){ }_{2} F_{1}\left(\begin{array}{c}
-n, n+\alpha+\frac{1}{2} \\
\frac{1}{2}
\end{array} \mid x^{2}\right), \\
n=0,1,2, \ldots,
\end{array}
$$

and

$$
P_{2 n+1}^{(\alpha, \alpha)}(x)=\left(-\frac{1}{4}\right)^{n}\left(\begin{array}{c}
2 n+\alpha \\
n
\end{array}\right)(2 n+\alpha+1) x_{2} F_{1}\left(\begin{array}{c}
-n, n+\alpha+\frac{3}{2} \mid x^{2} \\
\frac{3}{2} \\
n=0,1,2, \ldots
\end{array}\right.
$$

respectively. These formulas can be found in [3] in a slightly different notation.

We will often use the Vandermonde summation formula:

$$
{ }_{2} F_{1}\left(\begin{array}{c|c}
-n, b & 1 \\
c & 1
\end{array}\right)=\frac{(c-b)_{n}}{(c)_{n}}, \quad n=0,1,2, \ldots,
$$

and the Saalschütz summation formula: 


$$
\begin{array}{r}
{ }_{3} F_{2}\left(\begin{array}{c}
-n, a, b \\
c,-n+a+b-c+1
\end{array} \mid 1\right)=\frac{(c-a)_{n}(c-b)_{n}}{(c)_{n}(c-a-b)_{n}}, \\
n=0,1,2, \ldots
\end{array}
$$

These summation formulas can be found in [3] for instance.

Finally, we remark that the Taylor series at the point zero of a hypergeometric function of the form ${ }_{p+1} F_{p}$ has a radius of convergence 1 unless it terminates. Moreover, such a series also converges absolutely at 1 if the sum of the numerator parameters is less than the sum of the denominator parameters. For details the reader is referred to [1].

\section{THE DIFFERENTIAL EQUATIONS FOR $P_{n}^{\alpha, \alpha, M, M}(x)$}

In order to find all differential equations of the form

(11) $M \sum_{i=0}^{\infty} a_{i}(x) y^{(i)}(x)+\left(1-x^{2}\right) y^{\prime \prime}(x)-2(\alpha+1) x y^{\prime}(x)+n(n+2 \alpha+1) y(x)=0$

for the polynomials $\left\{P_{n}^{\alpha, \alpha, M, M}(x)\right\}_{n=0}^{\infty}$, where the coefficients $\left\{a_{i}(x)\right\}_{i=0}^{\infty}$ are continuous functions on the real line and where $\left\{a_{i}(x)\right\}_{i=1}^{\infty}$ are independent of $n$, we set $y(x)=P_{n}^{\alpha, \alpha, M, M}(x)$ in (11) and use the definition (1) to find

$$
\begin{aligned}
M C_{0} & \sum_{i=0}^{\infty} a_{i}(x) D^{i} P_{n}^{(\alpha, \alpha)}(x)-M C_{1} \sum_{i=0}^{\infty} i a_{i}(x) D^{i} P_{n}^{(\alpha, \alpha)}(x) \\
& -M C_{1} x \sum_{i=0}^{\infty} a_{i}(x) D^{i+1} P_{n}^{(\alpha, \alpha)}(x) \\
& +\left(1-x^{2}\right)\left[C_{0} \frac{d^{2}}{d x^{2}} P_{n}^{(\alpha, \alpha)}(x)-2 C_{1} \frac{d^{2}}{d x^{2}} P_{n}^{(\alpha, \alpha)}(x)-C_{1} x \frac{d^{3}}{d x^{3}} P_{n}^{(\alpha, \alpha)}(x)\right] \\
& -2(\alpha+1) x\left[C_{0} \frac{d}{d x} P_{n}^{(\alpha, \alpha)}(x)-C_{1} \frac{d}{d x} P_{n}^{(\alpha, \alpha)}(x)-C_{1} x \frac{d^{2}}{d x^{2}} P_{n}^{(\alpha, \alpha)}(x)\right] \\
& +n(n+2 \alpha+1)\left[C_{0} P_{n}^{(\alpha, \alpha)}(x)-C_{1} x \frac{d}{d x} P_{n}^{(\alpha, \alpha)}(x)\right]=0
\end{aligned}
$$

Now we use (6) with $i=0$ and $i=1$ to obtain

$$
\begin{aligned}
M C_{0} \sum_{i=0}^{\infty} a_{i}(x) D^{i} P_{n}^{(\alpha, \alpha)}(x) \\
\quad-M C_{1}\left[\sum_{i=0}^{\infty} i a_{i}(x) D^{i} P_{n}^{(\alpha, \alpha)}(x)+x \sum_{i=0}^{\infty} a_{i}(x) D^{i+1} P_{n}^{(\alpha, \alpha)}(x)\right] \\
=2 C_{1} \frac{d^{2}}{d x^{2}} P_{n}^{(\alpha, \alpha)}(x) .
\end{aligned}
$$


We consider both sides to be polynomials in $M$. Comparing the coefficients of equal powers of $M$ on both sides leads to

$$
\begin{gathered}
M: \sum_{i=0}^{\infty} a_{i}(x) D^{i} P_{n}^{(\alpha, \alpha)}(x)=\frac{4}{(2 \alpha+1)}\left(\begin{array}{c}
n+2 \alpha \\
n
\end{array}\right) \frac{d^{2}}{d x^{2}} P_{n}^{(\alpha, \alpha)}(x) \\
M^{2}: \frac{2 n}{(\alpha+1)}\left(\begin{array}{c}
n+2 \alpha+1 \\
n
\end{array}\right) \sum_{i=0}^{\infty} a_{i}(x) D^{i} P_{n}^{(\alpha, \alpha)}(x)-\frac{2}{(2 \alpha+1)}\left(\begin{array}{c}
n+2 \alpha \\
n
\end{array}\right) \\
\times\left[\sum_{i=0}^{\infty} i a_{i}(x) D^{i} P_{n}^{(\alpha, \alpha)}(x)+x \sum_{i=0}^{\infty} a_{i}(x) D^{i+1} P_{n}^{(\alpha, \alpha)}(x)\right] \\
=\frac{4}{(\alpha+1)}\left(\begin{array}{c}
n+2 \alpha \\
n-1
\end{array}\right)\left(\begin{array}{c}
n+2 \alpha+1 \\
n
\end{array}\right) \frac{d^{2}}{d x^{2}} P_{n}^{(\alpha, \alpha)}(x) \\
M^{3}: 4\left(\begin{array}{c}
n+2 \alpha+1 \\
n-1
\end{array}\right)^{2} \sum_{i=0}^{\infty} a_{i}(x) D^{i} P_{n}^{(\alpha, \alpha)}(x) \\
=\frac{2}{(\alpha+1)}\left(\begin{array}{c}
n+2 \alpha \\
n-1
\end{array}\right)\left(\begin{array}{c}
n+2 \alpha+1 \\
n
\end{array}\right) \\
\times\left[\sum_{i=0}^{\infty} i a_{i}(x) D^{i} P_{n}^{(\alpha, \alpha)}(x)+x \sum_{i=0}^{\infty} a_{i}(x) D^{i+1} P_{n}^{(\alpha, \alpha)}(x)\right] .
\end{gathered}
$$

It is clear that the third relation is a linear combination of the first two relations which can be simplified to

$$
\sum_{i=0}^{\infty} a_{i}(x) D^{i} P_{n}^{(\alpha, \alpha)}(x)=\frac{4}{(2 \alpha+1)}\left(\begin{array}{c}
n+2 \alpha \\
n
\end{array}\right) \frac{d^{2}}{d x^{2}} P_{n}^{(\alpha, \alpha)}(x)
$$

and

$$
\begin{gathered}
\sum_{i=0}^{\infty} i a_{i}(x) D^{i} P_{n}^{(\alpha, \alpha)}(x)+x \sum_{i=0}^{\infty} a_{i}(x) D^{i+1} P_{n}^{(\alpha, \alpha)}(x) \\
=4\left(\begin{array}{c}
n+2 \alpha+1 \\
n-1
\end{array}\right) \frac{d^{2}}{d x^{2}} P_{n}^{(\alpha, \alpha)}(x) .
\end{gathered}
$$

Since we demand that the coefficients $\left\{a_{i}(x)\right\}_{i=1}^{\infty}$ are independent of $n$, we introduce the following notation:

$$
\left\{\begin{array}{l}
a_{0}(x):=a_{0}(n, \alpha, x), \quad n=0,1,2, \ldots ; \\
a_{i}(x):=a_{i}(\alpha, x), \quad i=1,2,3, \ldots
\end{array}\right.
$$

In order to find the general form of the coefficients $\left\{a_{i}(x)\right\}_{i=0}^{\infty}$ we will prove the following theorems.

Theorem 1. The polynomials $\left\{P_{n}^{\alpha, \alpha, M, M}(x)\right\}_{n=0}^{\infty}$ satisfy the infinite-order differential equation given by

$$
\sum_{i=0}^{\infty} b_{i}(x) y^{(i)}(x)=0
$$

and

$$
\left\{\begin{array}{l}
b_{0}(x):=b_{0}(n, \alpha, x)=\frac{1}{2}\left[1-(-1)^{n}\right], \quad n=0,1,2, \ldots ; \\
b_{i}(x):=b_{i}(\alpha, x)=\frac{2^{i-1}}{i !}(-x)^{i}, \quad i=1,2,3, \ldots
\end{array}\right.
$$


Note that the coefficients $\left\{b_{i}(x)\right\}_{i=0}^{\infty}$ do not depend on $\alpha$. The proof of Theorem 1 can be found in the next section.

Theorem 2. The polynomials $\left\{P_{n}^{\alpha, \alpha, M, M}(x)\right\}_{n=0}^{\infty}$ satisfy the differential equation given by

$$
M \sum_{i=0}^{\infty} c_{i}(x) y^{(i)}(x)+\left(1-x^{2}\right) y^{\prime \prime}(x)-2(\alpha+1) x y^{\prime}(x)+n(n+2 \alpha+1) y(x)=0
$$

where the coefficients $\left\{c_{i}(x)\right\}_{i=0}^{\infty}$ are defined by

$$
c_{0}(x):=c_{0}(n, \alpha, x)=4(2 \alpha+3)\left(\begin{array}{c}
n+2 \alpha+2 \\
n-2
\end{array}\right), \quad n=0,1,2, \ldots,
$$

and

$$
c_{i}(x)=(2 \alpha+3)\left(1-x^{2}\right) c_{i}^{*}(x), \quad i=1,2,3, \ldots,
$$

where

$$
\left\{\begin{aligned}
& c_{1}^{*}(x):=c_{1}^{*}(\alpha, x)=0, \\
& c_{i}^{*}(x):=c_{i}^{*}(\alpha, x)=\frac{2 i}{i !} \sum_{k=0}^{i-2}\left(\begin{array}{c}
\alpha+1 \\
i-k-2
\end{array}\right)\left(\begin{array}{c}
i-2 \alpha-5 \\
k
\end{array}\right)\left(\frac{1-x}{2}\right)^{k}, \\
& i=2,3,4, \ldots .
\end{aligned}\right.
$$

The proof of Theorem 2 will be given in $\S 5$.

Now we will show that the general solution $\left\{a_{i}(x)\right\}_{i=0}^{\infty}$ of (12) and (13), where $\left\{a_{i}(x)\right\}_{i=0}^{\infty}$ are continuous functions on the real line and where $\left\{a_{i}(x)\right\}_{i=1}^{\infty}$ are independent of $n$, is

$$
\begin{cases}a_{0}(n, \alpha, x)=a_{0}(1, \alpha, x) b_{0}(n, \alpha, x)+c_{0}(n, \alpha, x), & n=0,1,2, \ldots, \\ a_{i}(\alpha, x)=a_{0}(1, \alpha, x) b_{i}(\alpha, x)+c_{i}(\alpha, x), \quad i=1,2,3, \ldots,\end{cases}
$$

where $a_{0}(1, \alpha, x)$ is an arbitrary continuous function on the real line and where the coefficients $\left\{b_{i}(x)\right\}_{i=0}^{\infty}$ and $\left\{c_{i}(x)\right\}_{i=0}^{\infty}$ are given by (16), (18), (19), and (20).

The proof is based on the following lemma.

Lemma. Let $\left\{a_{i}(x)\right\}_{i=0}^{\infty}$ be a set of continuous functions of the form (14) which satisfies the homogeneous system

$$
\left\{\begin{array}{l}
\sum_{i=0}^{\infty} a_{i}(x) D^{i} P_{n}^{(\alpha, \alpha)}(x)=0 \\
\sum_{i=0}^{\infty} i a_{i}(x) D^{i} P_{n}^{(\alpha, \alpha)}(x)+x \sum_{i=0}^{\infty} a_{i}(x) D^{i+1} P_{n}^{(\alpha, \alpha)}(x)=0
\end{array}\right.
$$

with $a_{0}(1, \alpha, x)=0$ for all real $x$. Then we have

$$
a_{i}(x)=0, \quad i=0,1,2, \ldots,
$$

for all real $x$.

In order to prove this lemma we substitute small values of $n$ in the homogeneous system (22). Then we obtain for $n=0$ and $n=1$ :

$$
a_{0}(0, \alpha, x)=0 \quad \text { and } \quad(\alpha+1)\left[x a_{0}(1, \alpha, x)+a_{1}(\alpha, x)\right]=0
$$


for all real $x$. Since $a_{0}(1, \alpha, x)=0$ and $\alpha>-1$, we conclude that $a_{1}(\alpha, x)=$ 0 for all real $x$. If we substitute $n=2$ in (22), we obtain

Hence

$$
\left\{\begin{array}{l}
a_{0}(2, \alpha, x) P_{2}^{(\alpha, \alpha)}(x)+a_{2}(\alpha, x) \frac{d^{2}}{d x^{2}} P_{2}^{(\alpha, \alpha)}(x)=0, \\
2 a_{2}(\alpha, x) \frac{d^{2}}{d x^{2}} P_{2}^{(\alpha, \alpha)}(x)+x a_{0}(2, \alpha, x) \frac{d}{d x} P_{2}^{(\alpha, \alpha)}(x)=0 .
\end{array}\right.
$$

Since

$$
\left\{\begin{array}{l}
{\left[2 P_{2}^{(\alpha, \alpha)}(x)-x \frac{d}{d x} P_{2}^{(\alpha, \alpha)}(x)\right] a_{0}(2, \alpha, x)=0} \\
a_{2}(\alpha, x) \frac{d^{2}}{d x^{2}} P_{2}^{(\alpha, \alpha)}(x)=-a_{0}(2, \alpha, x) P_{2}^{(\alpha, \alpha)}(x)
\end{array}\right.
$$

$$
2 P_{2}^{(\alpha, \alpha)}(x)-x \frac{d}{d x} P_{2}^{(\alpha, \alpha)}(x)=-\frac{1}{2}(\alpha+2) \neq 0
$$

we conclude that $a_{0}(2, \alpha, x)=0$ for all real $x$ and therefore that $a_{2}(\alpha, x)=0$ for all real $x$, since

$$
\frac{d^{2}}{d x^{2}} P_{2}^{(\alpha, \alpha)}(x)=\frac{1}{2}(\alpha+2)(2 \alpha+3) \neq 0
$$

In the same way we obtain for $n=3$ :

$$
\left\{\begin{array}{l}
{\left[3 P_{3}^{(\alpha, \alpha)}(x)-x \frac{d}{d x} P_{3}^{(\alpha, \alpha)}(x)\right] a_{0}(3, \alpha, x)=0} \\
a_{3}(\alpha, x) \frac{d^{3}}{d x^{3}} P_{3}^{(\alpha, \alpha)}(x)=-a_{0}(3, \alpha, x) P_{3}^{(\alpha, \alpha)}(x)
\end{array}\right.
$$

Now we find $a_{0}(3, \alpha, x)=0$ for all real $x$ except for $x=0$ being the only zero of

$$
3 P_{3}^{(\alpha, \alpha)}(x)-x \frac{d}{d x} P_{3}^{(\alpha, \alpha)}(x)=-\frac{1}{2}(\alpha+2)(\alpha+3) x .
$$

The continuity of $a_{0}(3, \alpha, x)$ implies that $a_{0}(3, \alpha, x)=0$ for all real $x$. Then we also have $a_{3}(\alpha, x)=0$ for all real $x$, since

$$
\frac{d^{3}}{d x^{3}} P_{3}^{(\alpha, \alpha)}(x)=\frac{1}{2}(\alpha+2)(\alpha+3)(2 \alpha+5) \neq 0
$$

If we proceed in this way we also find for each $n \geq 4$ that $a_{0}(n, \alpha, x)=0$ for all real $x$ except for the possible zeros of $n P_{n}^{(\alpha, \alpha)}(x)-x \frac{d}{d x} P_{n}^{(\alpha, \alpha)}(x)$. The continuity of $a_{0}(n, \alpha, x)$ then implies that $a_{0}(n, \alpha, x)=0$ for all real $x$, and finally we have $a_{n}(\alpha, x)=0$ for all real $x$, since

$$
\frac{d^{n}}{d x^{n}} P_{n}^{(\alpha, \alpha)}(x)=\left(\begin{array}{c}
2 n+2 \alpha \\
n
\end{array}\right) \frac{n !}{2^{n}} \neq 0 \text {. }
$$

This completes the proof of the lemma.

For the moment we introduce the term continuous sequence. A sequence $\left\{a_{i}(x)\right\}_{i=0}^{\infty}$ is called a continuous sequence if $\left\{a_{i}(x)\right\}_{i=0}^{\infty}$ are continuous functions on the real line of the form (14).

In $\S 4$ (proof of Theorem 1) we show that the continuous sequence $\left\{b_{i}(x)\right\}_{i=0}^{\infty}$ defined by (16) is a solution of the homogeneous system (22). This implies that the general solution $\left\{a_{i}(x)\right\}_{i=0}^{\infty}$ of (22), being a continuous sequence, is

$$
a_{i}(x)=a_{0}(1, \alpha, x) b_{i}(x), \quad i=0,1,2, \ldots
$$


This can be shown by using the lemma as follows. We define

$$
a_{i}^{*}(x):=a_{i}(x)-a_{0}(1, \alpha, x) b_{i}(x), \quad i=0,1,2, \ldots
$$

Since $a_{0}(1, \alpha, x)$ is continuous, $\left\{a_{i}^{*}(x)\right\}_{i=0}^{\infty}$ is a continuous sequence which is a solution of $(22)$ too where

$$
a_{0}^{*}(1, \alpha, x)=a_{0}(1, \alpha, x)-a_{0}(1, \alpha, x) b_{0}(1, \alpha, x)=0
$$

for all real $x$. Now the lemma gives us the desired result.

In $\S 5$ (proof of Theorem 2) we show that the continuous sequence $\left\{c_{i}(x)\right\}_{i=0}^{\infty}$ given by (18), (19), and (20) is a solution of (12) and (13). In order to prove that the general solution $\left\{a_{i}(x)\right\}_{i=0}^{\infty}$ of (12) and (13), being a continuous sequence, is of the form (21), we simply note that $\left\{a_{i}(x)-c_{i}(x)\right\}_{i=0}^{\infty}$ is a continuous sequence and a solution of the homogeneous system (22). Hence

$$
a_{i}(x)-c_{i}(x)=a_{0}(1, \alpha, x) b_{i}(x), \quad i=0,1,2, \ldots
$$

This shows that we have found all differential equations of the form (11), where the coefficients $\left\{a_{i}(x)\right\}_{i=0}^{\infty}$ are continuous functions on the real line and where $\left\{a_{i}(x)\right\}_{i=1}^{\infty}$ are independent of $n$.

In [6] we conjectured that the polynomials $\left\{P_{n}^{\alpha, \alpha, M, M}(x)\right\}_{n=0}^{\infty}$ satisfy a differential equation of the form (17) where the coefficients $\left\{c_{i}(x)\right\}_{i=0}^{\infty}$ are given by (18) and (19) where

$$
\left\{\begin{array}{l}
c_{2 i}^{*}(\alpha, x)=\frac{4(-1)^{i+1}}{(2 i) !}\left(\begin{array}{c}
\alpha+1 \\
i-1
\end{array}\right){ }_{2} F_{1}\left(\begin{array}{c}
-i+1, \alpha+\frac{5}{2}-i \\
\frac{1}{2}
\end{array} \mid x^{2}\right), \\
i=1,2,3, \ldots \\
c_{1}^{*}(\alpha, x)=0 ; \\
c_{2 i+1}^{*}(\alpha, x)=\frac{8(-1)^{i+1}}{(2 i+1) !}\left(\begin{array}{c}
\alpha \\
i-1
\end{array}\right)(\alpha+1) x_{2} F_{1}\left(\begin{array}{r}
-i+1, \alpha+\frac{5}{2}-i \mid \\
\left.\frac{3}{2} \quad x^{2}\right), \\
i=1,2,3, \ldots
\end{array}\right.
\end{array}\right.
$$

By using the formulas (7) and (8) we can prove that (23) is equivalent to

$$
\left\{\begin{array}{l}
c_{1}^{*}(\alpha, x)=0 \\
c_{i}^{*}(\alpha, x)=\frac{2^{i}}{i !} P_{i-2}^{(\alpha-i+3, \alpha-i+3)}(x), \quad i=2,3,4, \ldots
\end{array}\right.
$$

Here we remark that the parameters $\alpha-i+3$ in the Jacobi polynomial might be smaller than -1 . However, the ultraspherical polynomial $P_{n}^{(\alpha, \alpha)}(x)$ is also a polynomial in $\alpha$. Instead of the definition (3) we may define

$$
\begin{aligned}
& P_{n}^{(\alpha, \alpha)}(x)=\frac{1}{n !} \sum_{k=0}^{n}\left(\begin{array}{l}
n \\
k
\end{array}\right)(n+2 \alpha+1)_{k}(\alpha+k+1)_{n-k}\left(\frac{x-1}{2}\right)^{k}, \\
& n=0,1,2, \ldots
\end{aligned}
$$

By using this definition of the ultraspherical polynomial we see that (24) is equivalent to $(20)$.

Since by using (21), (16), (19), and (23) we have

$$
a_{2 i}(0):=a_{2 i}(\alpha, 0)=(2 \alpha+3) \frac{4(-1)^{i+1}}{(2 i) !}\left(\begin{array}{c}
\alpha+1 \\
i-1
\end{array}\right) \neq 0, \quad i=1,2,3, \ldots,
$$


if $\alpha$ is not a nonnegative integer, we conclude that if $M>0$ the order of the differential equation given by (11) can only be finite for nonnegative integer values of $\alpha$. From the form (23) we easily see that for nonnegative integer values of $\alpha$ we have

$$
\begin{cases}\text { degree }\left[c_{i}(x)\right]=i, & i=2,3,4, \ldots, 2 \alpha+4 \\ c_{i}(x)=0, & i>2 \alpha+4\end{cases}
$$

Hence, for $M>0$ the order of the differential equation given by (11) can only be finite if we choose $a_{0}(1, \alpha, x)=0$ in view of (21) and if $\alpha$ is a nonnegative integer. In that case the order of the differential equation given by (17), (18), (19), and (20) equals $2 \alpha+4$.

\section{Proof of Theorem 1}

To prove Theorem 1 we have to show that

$$
\left\{\begin{array}{l}
\sum_{i=0}^{\infty} b_{i}(x) D^{i} P_{n}^{(\alpha, \alpha)}(x)=0 \\
\sum_{i=0}^{\infty} i b_{i}(x) D^{i} P_{n}^{(\alpha, \alpha)}(x)+x \sum_{i=0}^{\infty} b_{i}(x) D^{i+1} P_{n}^{(\alpha, \alpha)}(x)=0 .
\end{array}\right.
$$

To do this we first note that we have in view of (16)

$$
\sum_{i=0}^{\infty} b_{i}(x) D^{i} P_{n}^{(\alpha, \alpha)}(x)=\frac{1}{2}\left[1-(-1)^{n}\right] P_{n}^{(\alpha, \alpha)}(x)+\sum_{i=1}^{\infty} b_{i}(x) D^{i} P_{n}^{(\alpha, \alpha)}(x)
$$

and

$$
\sum_{i=0}^{\infty} b_{i}(x) D^{i+1} P_{n}^{(\alpha, \alpha)}(x)=\frac{1}{2}\left[1-(-1)^{n}\right] \frac{d}{d x} P_{n}^{(\alpha, \alpha)}(x)+\sum_{i=1}^{\infty} b_{i}(x) D^{i+1} P_{n}^{(\alpha, \alpha)}(x) .
$$

Now we use (4), (5), and (16) to obtain

$$
\begin{aligned}
\sum_{i=1}^{\infty} b_{i}(x) D^{i} P_{n}^{(\alpha, \alpha)}(x) \\
=\frac{1}{2}\left(\begin{array}{c}
n+\alpha \\
n
\end{array}\right) \sum_{i=1}^{\infty} \frac{x^{i}}{i !} \sum_{k=i}^{\infty} \frac{(-n)_{k}(n+2 \alpha+1)_{k}}{(\alpha+1)_{k}(k-i) !}\left(\frac{1-x}{2}\right)^{k-i} \\
=\frac{1}{2}\left(\begin{array}{c}
n+\alpha \\
n
\end{array}\right) \sum_{k=1}^{\infty} \frac{(-n)_{k}(n+2 \alpha+1)_{k}}{(\alpha+1)_{k} k !} \sum_{i=1}^{k}\left(\begin{array}{c}
k \\
i
\end{array}\right) x^{i}\left(\frac{1-x}{2}\right)^{k-i} \\
=\frac{1}{2}\left(\begin{array}{c}
n+\alpha \\
n
\end{array}\right) \sum_{k=1}^{\infty} \frac{(-n)_{k}(n+2 \alpha+1)_{k}}{(\alpha+1)_{k} k !}\left[\left(\frac{1+x}{2}\right)^{k}-\left(\frac{1-x}{2}\right)^{k}\right] \\
=\frac{1}{2}\left[P_{n}^{(\alpha, \alpha)}(-x)-P_{n}^{(\alpha, \alpha)}(x)\right]=-\frac{1}{2}\left[1-(-1)^{n}\right] P_{n}^{(\alpha, \alpha)}(x),
\end{aligned}
$$




$$
\begin{aligned}
\sum_{i=0}^{\infty} i b_{i}(x) D^{i} P_{n}^{(\alpha, \alpha)}(x) \\
=\frac{1}{2}\left(\begin{array}{c}
n+\alpha \\
n
\end{array}\right) \sum_{i=1}^{\infty} \frac{x^{i}}{(i-1) !} \sum_{k=i}^{\infty} \frac{(-n)_{k}(n+2 \alpha+1)_{k}}{(\alpha+1)_{k}(k-i) !}\left(\frac{1-x}{2}\right)^{k-i} \\
=\frac{1}{2}\left(\begin{array}{c}
n+\alpha \\
n
\end{array}\right) \sum_{k=1}^{\infty} \frac{(-n)_{k}(n+2 \alpha+1)_{k}}{(\alpha+1)_{k}(k-1) !} \sum_{i=1}^{k}\left(\begin{array}{c}
k-1 \\
i-1
\end{array}\right) x^{i}\left(\frac{1-x}{2}\right)^{k-i} \\
=\frac{1}{2}\left(\begin{array}{c}
n+\alpha \\
n
\end{array}\right) x \sum_{k=1}^{\infty} \frac{(-n)_{k}(n+2 \alpha+1)_{k}}{(\alpha+1)_{k}(k-1) !}\left(\frac{1+x}{2}\right)^{k-1} \\
=(-1)^{n} x \frac{d}{d x} P_{n}^{(\alpha, \alpha)}(x)
\end{aligned}
$$

and

$$
\begin{aligned}
\sum_{i=1}^{\infty} b_{i}(x) D^{i+1} P_{n}^{(\alpha, \alpha)}(x) \\
=-\frac{1}{4}\left(\begin{array}{c}
n+\alpha \\
n
\end{array}\right) \sum_{i=1}^{\infty} \frac{x^{i}}{i !} \sum_{k=i+1}^{\infty} \frac{(-n)_{k}(n+2 \alpha+1)_{k}}{(\alpha+1)_{k}(k-i-1) !}\left(\frac{1-x}{2}\right)^{k-i-1} \\
=-\frac{1}{4}\left(\begin{array}{c}
n+\alpha \\
n
\end{array}\right) \sum_{k=2}^{\infty} \frac{(-n)_{k}(n+2 \alpha+1)_{k}}{(\alpha+1)_{k}(k-1) !} \sum_{i=1}^{k-1}\left(\begin{array}{c}
k-1 \\
i
\end{array}\right) x^{i}\left(\frac{1-x}{2}\right)^{k-i-1} \\
=-\frac{1}{4}\left(\begin{array}{c}
n+\alpha \\
n
\end{array}\right) \sum_{k=2}^{\infty} \frac{(-n)_{k}(n+2 \alpha+1)_{k}}{(\alpha+1)_{k}(k-1) !}\left[\left(\frac{1+x}{2}\right)^{k-1}-\left(\frac{1-x}{2}\right)^{k-1}\right] \\
=-\frac{1}{2}\left[1+(-1)^{n}\right] \frac{d}{d x} P_{n}^{(\alpha, \alpha)}(x) .
\end{aligned}
$$

This proves Theorem 1.

\section{Proof of Theorem 2}

In this section we will give a proof of the main Theorem 2 .

In view of (12) and (13) we have to show that

$$
\sum_{i=0}^{\infty} c_{i}(x) D^{i} P_{n}^{(\alpha, \alpha)}(x)=\frac{4}{(2 \alpha+1)}\left(\begin{array}{c}
n+2 \alpha \\
n
\end{array}\right) \frac{d^{2}}{d x^{2}} P_{n}^{(\alpha, \alpha)}(x)
$$

and

$$
\begin{aligned}
& \sum_{i=0}^{\infty} i c_{i}(x) D^{i} P_{n}^{(\alpha, \alpha)}(x)+x \sum_{i=0}^{\infty} c_{i}(x) D^{i+1} P_{n}^{(\alpha, \alpha)}(x) \\
& =4\left(\begin{array}{c}
n+2 \alpha+1 \\
n-1
\end{array}\right) \frac{d^{2}}{d x^{2}} P_{n}^{(\alpha, \alpha)}(x)
\end{aligned}
$$


Now we write by using (18), (19), and (20)

$$
\begin{aligned}
\sum_{i=0}^{\infty} c_{i}(x) D^{i} P_{n}^{(\alpha, \alpha)}(x) & \\
= & 4(2 \alpha+3)\left(\begin{array}{c}
n+2 \alpha+2 \\
n-2
\end{array}\right) P_{n}^{(\alpha, \alpha)}(x)+\sum_{i=2}^{\infty} c_{i}(x) D^{i} P_{n}^{(\alpha, \alpha)}(x) \\
& \sum_{i=0}^{\infty} i c_{i}(x) D^{i} P_{n}^{(\alpha, \alpha)}(x)=\sum_{i=2}^{\infty} i c_{i}(x) D^{i} P_{n}^{(\alpha, \alpha)}(x)
\end{aligned}
$$

and

$$
\begin{aligned}
\sum_{i=0}^{\infty} c_{i}(x) D^{i+1} P_{n}^{(\alpha, \alpha)}(x)= & 4(2 \alpha+3)\left(\begin{array}{c}
n+2 \alpha+2 \\
n-2
\end{array}\right) \frac{d}{d x} P_{n}^{(\alpha, \alpha)}(x) \\
& +\sum_{i=2}^{\infty} c_{i}(x) D^{i+1} P_{n}^{(\alpha, \alpha)}(x) .
\end{aligned}
$$

Now we obtain by using (20)

$$
\begin{aligned}
& \sum_{i=2}^{\infty} c_{i}^{*}(x) D^{i} P_{n}^{(\alpha, \alpha)}(x) \\
&=\sum_{i=2}^{\infty} \frac{2^{i}}{i !} \sum_{k=0}^{i-2}\left(\begin{array}{c}
\alpha+1 \\
i-k-2
\end{array}\right)\left(\begin{array}{c}
i-2 \alpha-5 \\
k
\end{array}\right)\left(\frac{1-x}{2}\right)^{k} D^{i} P_{n}^{(\alpha, \alpha)}(x) \\
&=\sum_{k=0}^{\infty} \sum_{i=k+2}^{\infty} \frac{2 i}{i !}\left(\begin{array}{c}
\alpha+1 \\
i-k-2
\end{array}\right)\left(\begin{array}{c}
i-2 \alpha-5 \\
k
\end{array}\right)\left(\frac{1-x}{2}\right)^{k} D^{i} P_{n}^{(\alpha, \alpha)}(x) \\
&= \sum_{k=0}^{\infty} \sum_{i=0}^{\infty} \frac{2^{i+k+2}}{(i+k+2) !}\left(\begin{array}{c}
\alpha+1 \\
i
\end{array}\right)\left(\begin{array}{c}
i+k-2 \alpha-3 \\
k
\end{array}\right)\left(\frac{1-x}{2}\right)^{k} \\
& \times D^{i+k+2} P_{n}^{(\alpha, \alpha)}(x) \\
&= \sum_{i=0}^{\infty}\left(\begin{array}{c}
\alpha+1 \\
i
\end{array}\right) \frac{2^{i+2}}{(i+2) !} \sum_{k=0}^{\infty} \frac{(-2 \alpha+i-2)_{k}}{k !(i+3)_{k}}(1-x)^{k} D^{i+k+2} P_{n}^{(\alpha, \alpha)}(x) .
\end{aligned}
$$

By using (4) we find

$$
\begin{aligned}
D^{i+k+2} & P_{n}^{(\alpha, \alpha)}(x) \\
= & \left(\begin{array}{c}
n+\alpha \\
n
\end{array}\right)\left(-\frac{1}{2}\right)^{i+k+2} \\
& \times \sum_{m=i+k+2}^{\infty} \frac{(-n)_{m}(n+2 \alpha+1)_{m}}{(m-i-k-2) !(\alpha+1)_{m}}\left(\frac{1-x}{2}\right)^{m-i-k-2} \\
= & \left(\begin{array}{c}
n+\alpha \\
n
\end{array}\right)\left(-\frac{1}{2}\right)^{i+k+2} \sum_{m=k}^{\infty} \frac{(-n)_{m+i+2}(n+2 \alpha+1)_{m+i+2}}{(m-k) !(\alpha+1)_{m+i+2}}\left(\frac{1-x}{2}\right)^{m-k} .
\end{aligned}
$$


Now we use Vandermonde's summation formula (9) to see that this leads to

$$
\begin{aligned}
& \sum_{k=0}^{\infty} \frac{(-2 \alpha+i-2)_{k}}{k !(i+3)_{k}}(1-x)^{k} D^{i+k+2} P_{n}^{(\alpha, \alpha)}(x) \\
& =\left(\begin{array}{c}
n+\alpha \\
n
\end{array}\right) \sum_{k=0}^{\infty} \sum_{m=k}^{\infty} \frac{(-2 \alpha+i-2)_{k}}{k !(i+3)_{k}} \\
& \times \frac{(-n)_{m+i+2}(n+2 \alpha+1)_{m+i+2}}{(m-k) !(\alpha+1)_{m+i+2}}(-1)^{i+k} \frac{(1-x)^{m}}{2^{i+m+2}} \\
& =\left(\begin{array}{c}
n+\alpha \\
n
\end{array}\right) \sum_{m=0}^{\infty} \sum_{k=0}^{m} \frac{(-2 \alpha+i-2)_{k}}{k !(i+3)_{k}} \\
& \times \frac{(-n)_{m+i+2}(n+2 \alpha+1)_{m+i+2}}{2^{m+i+2}(\alpha+1)_{m+i+2}} \frac{(-m)_{k}}{m !}(-1)^{i}(1-x)^{m} \\
& =\left(\begin{array}{c}
n+\alpha \\
n
\end{array}\right)(-1)^{i} \sum_{m=0}^{\infty} \frac{(-n)_{m+i+2}(n+2 \alpha+1)_{m+i+2}}{2^{i+m+2}(\alpha+1)_{m+i+2}} \frac{(1-x)^{m}}{m !} \\
& \times{ }_{2} F_{1}\left(\begin{array}{c|c}
-m,-2 \alpha+i-2 & 1 \\
i+3 & 1
\end{array}\right) \\
& =\left(\begin{array}{c}
n+\alpha \\
n
\end{array}\right)(-1)^{i} \sum_{m=0}^{\infty} \frac{(-n)_{m+i+2}(n+2 \alpha+1)_{m+i+2}}{2^{m+i+2}(\alpha+1)_{m+i+2}} \frac{(1-x)^{m}}{m !} \frac{(2 \alpha+5)_{m}}{(i+3)_{m}} \\
& =\left(\begin{array}{c}
n+\alpha \\
n
\end{array}\right)(-1)^{i} \frac{(i+2) !}{2^{i+2}} \\
& \times \sum_{m=0}^{\infty} \frac{(-n)_{m+i+2}(n+2 \alpha+1)_{m+i+2}}{(m+i+2) !(\alpha+1)_{m+i+2}} \frac{(2 \alpha+5)_{m}}{m !}\left(\frac{1-x}{2}\right)^{m} .
\end{aligned}
$$

\section{Hence}

$$
\begin{aligned}
\sum_{i=2}^{\infty} c_{i}^{*}(x) D^{i} P_{n}^{(\alpha, \alpha)}(x) \\
=\sum_{i=0}^{\infty}\left(\begin{array}{c}
\alpha+1 \\
i
\end{array}\right) \frac{2^{i+2}}{(i+2) !} \sum_{k=0}^{\infty} \frac{(-2 \alpha+i-2)_{k}}{k !(i+3)_{k}}(1-x)^{k} D^{i+k+2} P_{n}^{(\alpha, \alpha)}(x) \\
=\left(\begin{array}{c}
n+\alpha \\
n
\end{array}\right) \sum_{i=0}^{\infty}(-1)^{i}\left(\begin{array}{c}
\alpha+1 \\
i
\end{array}\right) \\
\quad \times \sum_{m=0}^{\infty} \frac{(-n)_{m+i+2}(n+2 \alpha+1)_{m+i+2}}{(m+i+2) !(\alpha+1)_{m+i+2}} \frac{(2 \alpha+5)_{m}}{m !}\left(\frac{1-x}{2}\right)^{m} \\
=\left(\begin{array}{c}
n+\alpha \\
n
\end{array}\right) \sum_{m=0}^{\infty} \frac{(-n)_{m+2}(n+2 \alpha+1)_{m+2}}{(m+2) !(\alpha+1)_{m+2}} \frac{(2 \alpha+5)_{m}}{m !}\left(\frac{1-x}{2}\right)^{m} \\
\quad \times{ }_{3} F_{2}\left(\begin{array}{c}
-n+m+2,-\alpha-1, n+2 \alpha+m+3 \\
m+3, \alpha+m+3
\end{array}\right) .
\end{aligned}
$$


Now we use the well-known fact that

$$
\begin{aligned}
{ }_{3} F_{2}\left(\begin{array}{c}
a, b, c \\
d, e
\end{array} \mid z\right)= & \frac{c}{c-a}{ }_{3} F_{2}\left(\begin{array}{c}
a, b, c+1 \\
d, e
\end{array}\right) \\
& -\frac{a}{c-a}{ }_{3} F_{2}\left(\begin{array}{c}
a+1, b, c \mid z \\
d, e
\end{array}\right), \quad c-a \neq 0
\end{aligned}
$$

and the Saalschütz summation formula (10) to find for $n>m+2$

$$
\begin{aligned}
&{ }_{3} F_{2}\left(\begin{array}{c}
-n+m+2,-\alpha-1, n+2 \alpha+m+3 \mid \\
m+3, \alpha+m+3
\end{array}\right) \\
&=\frac{(n+2 \alpha+m+3)}{(2 n+2 \alpha+1)}{ }_{3} F_{2}\left(\begin{array}{c}
-n+m+2,-\alpha-1, n+2 \alpha+m+4 \mid 1 \\
m+3, \alpha+m+3
\end{array}\right) \\
& \quad+\frac{(n-m-2)}{(2 n+2 \alpha+1)}{ }_{3} F_{2}\left(\begin{array}{c}
-n+m+3,-\alpha-1, n+2 \alpha+m+3 \mid \\
m+3, \alpha+m+3
\end{array}\right) \\
&=\frac{(n+2 \alpha+m+3)}{(2 n+2 \alpha+1)} \frac{(\alpha+m+4)_{n-m-2}(-n-2 \alpha-1)_{n-m-2}}{(m+3)_{n-m-2}(-n-\alpha)_{n-m-2}} \\
& \quad+\frac{(n-m-2)}{(2 n+2 \alpha+1)} \frac{(\alpha+m+4)_{n-m-3}(-n-2 \alpha)_{n-m-3}}{(m+3)_{n-m-3}(-n-\alpha+1)_{n-m-3}} \\
&= \frac{\Gamma(n+2 \alpha+1)}{n !} \frac{1}{(\alpha+m+3)} \frac{(m+2) !}{\Gamma(m+2 \alpha+4)} \\
& \times[n(n+2 \alpha+1)+(\alpha+1)(2 \alpha+m+3)] .
\end{aligned}
$$

Note that the same result also holds for $n=m+2$. For $n<m+2$ we have $(-n)_{m+2}=0$ and the ${ }_{3} F_{2}(1)$ exists since

$$
(-n+m+2)+(-\alpha-1)+(n+2 \alpha+m+3)<(m+3)+(\alpha+i n+3) .
$$

This implies that

$$
\begin{aligned}
& \sum_{i=2}^{\infty} c_{i}^{*}(x) D^{i} P_{n}^{(\alpha, \alpha)}(x) \\
&=\left(\begin{array}{c}
n+\alpha \\
n
\end{array}\right) \frac{\Gamma(n+2 \alpha+1)}{n !} \sum_{m=0}^{\infty} \frac{(-n)_{m+2}(n+2 \alpha+1)_{m+2}}{m !(\alpha+1)_{m+3}} \frac{(2 \alpha+5)_{m}}{\Gamma(m+2 \alpha+4)} \\
& \quad \times[n(n+2 \alpha+1)+(\alpha+1)(2 \alpha+m+3)]\left(\frac{1-x}{2}\right)^{m} \\
&=\frac{1}{(2 \alpha+1)_{4}}\left(\begin{array}{c}
n+\alpha \\
n
\end{array}\right)\left(\begin{array}{c}
n+2 \alpha \\
n
\end{array}\right) \\
& \times \sum_{m=0}^{\infty} \frac{(-n)_{m+2}(n+2 \alpha+1)_{m+2}}{m !(\alpha+1)_{m+3}}(m+2 \alpha+4) \\
& \times[n(n+2 \alpha+1)+(\alpha+1)(m+2 \alpha+3)]\left(\frac{1-x}{2}\right)^{m} .
\end{aligned}
$$


Since we have

$$
\begin{aligned}
& n(n+2 \alpha+1)+(\alpha+1)(m+2 \alpha+3) \\
& \quad=(\alpha+m+3)(m+2 \alpha+3)+(n-m-2)(n+2 \alpha+m+3)
\end{aligned}
$$

and

$$
(m+2 \alpha+3)(m+2 \alpha+4)=m(m-1)+2(2 \alpha+4) m+(2 \alpha+3)(2 \alpha+4)
$$

we obtain

$$
\begin{aligned}
& \sum_{i=2}^{\infty} c_{i}^{*}(x) D^{i} P_{n}^{(\alpha, \alpha)}(x) \\
& =\frac{1}{(2 \alpha+1)_{4}}\left(\begin{array}{c}
n+\alpha \\
n
\end{array}\right)\left(\begin{array}{c}
n+2 \alpha \\
n
\end{array}\right) \\
& \times\left[\sum_{m=0}^{\infty} \frac{(-n)_{m+4}(n+2 \alpha+1)_{m+4}}{m !(\alpha+1)_{m+4}}\left(\frac{1-x}{2}\right)^{m+2}\right. \\
& +2(2 \alpha+4) \sum_{m=0}^{\infty} \frac{(-n)_{m+3}(n+2 \alpha+1)_{m+3}}{m !(\alpha+1)_{m+3}}\left(\frac{1-x}{2}\right)^{m+1} \\
& +(2 \alpha+3)(2 \alpha+4) \sum_{m=0}^{\infty} \frac{(-n)_{m+2}(n+2 \alpha+1)_{m+2}}{m !(\alpha+1)_{m+2}}\left(\frac{1-x}{2}\right)^{m} \\
& -\sum_{m=0}^{\infty} \frac{(-n)_{m+4}(n+2 \alpha+1)_{m+4}}{m !(\alpha+1)_{m+4}}\left(\frac{1-x}{2}\right)^{m+1} \\
& \left.-(2 \alpha+4) \sum_{m=0}^{\infty} \frac{(-n)_{m+3}(n+2 \alpha+1)_{m+3}}{m !(\alpha+1)_{m+3}}\left(\frac{1-x}{2}\right)^{m}\right] \\
& =\frac{4}{(2 \alpha+1)_{4}}\left(\begin{array}{c}
n+2 \alpha \\
n
\end{array}\right) \\
& \times\left[4\left(\frac{1-x}{2}\right)^{2} \frac{d^{4}}{d x^{4}} P_{n}^{(\alpha, \alpha)}(x)\right. \\
& -4(2 \alpha+4)\left(\frac{1-x}{2}\right) \frac{d^{3}}{d x^{3}} P_{n}^{(\alpha, \alpha)}(x) \\
& +(2 \alpha+3)(2 \alpha+4) \frac{d^{2}}{d x^{2}} P_{n}^{(\alpha, \alpha)}(x) \\
& \left.-4\left(\frac{1-x}{2}\right) \frac{d^{4}}{d x^{4}} P_{n}^{(\alpha, \alpha)}(x)+2(2 \alpha+4) \frac{d^{3}}{d x^{3}} P_{n}^{(\alpha, \alpha)}(x)\right] \\
& =\frac{4}{(2 \alpha+1)_{4}}\left(\begin{array}{c}
n+2 \alpha \\
n
\end{array}\right) \\
& \times\left[-\left(1-x^{2}\right) \frac{d^{4}}{d x^{4}} P_{n}^{(\alpha, \alpha)}(x)+2(2 \alpha+4) x \frac{d^{3}}{d x^{3}} P_{n}^{(\alpha, \alpha)}(x)\right. \\
& \left.+(2 \alpha+3)(2 \alpha+4) \frac{d^{2}}{d x^{2}} P_{n}^{(\alpha, \alpha)}(x)\right] .
\end{aligned}
$$


Now we use (6) for $i=2$ to find

$$
\begin{aligned}
& \sum_{i=2}^{\infty} c_{i}^{*}(x) D^{i} P_{n}^{(\alpha, \alpha)}(x)=\frac{4}{(2 \alpha+1)_{4}}\left(\begin{array}{c}
n+2 \alpha \\
n
\end{array}\right) \\
& \times\left[2(\alpha+1) x \frac{d^{3}}{d x^{3}} P_{n}^{(\alpha, \alpha)}(x)\right. \\
& \left.\quad+[(n-2)(n+2 \alpha+3)+(2 \alpha+3)(2 \alpha+4)] \frac{d^{2}}{d x^{2}} P_{n}^{(\alpha, \alpha)}(x)\right] .
\end{aligned}
$$

Hence, by using (19) and (6) for $i=1$ and $i=0$ we find

$$
\begin{aligned}
& \sum_{i=2}^{\infty} c_{i}(x) D^{i} P_{n}^{(\alpha, \alpha)}(x) \\
& =\frac{1}{(\alpha+1)(\alpha+2)(2 \alpha+1)}\left(\begin{array}{c}
n+2 \alpha \\
n
\end{array}\right)\left(1-x^{2}\right) \\
& \times\left[2(\alpha+1) x \frac{d^{3}}{d x^{3}} P_{n}^{(\alpha, \alpha)}(x)\right. \\
& \left.+[(n-2)(n+2 \alpha+3)+(2 \alpha+3)(2 \alpha+4)] \frac{d^{2}}{d x^{2}} P_{n}^{(\alpha, \alpha)}(x)\right] \\
& =\frac{1}{(\alpha+1)(\alpha+2)(2 \alpha+1)}\left(\begin{array}{c}
n+2 \alpha \\
n
\end{array}\right) \\
& \times\left[4(\alpha+1)(\alpha+2) x^{2} \frac{d^{2}}{d x^{2}} P_{n}^{(\alpha, \alpha)}(x)\right. \\
& -(n-1)(n+2 \alpha+2)\left(1-x^{2}\right) \frac{d^{2}}{d x^{2}} P_{n}^{(\alpha, \alpha)}(x) \\
& -n(n-1)(n+2 \alpha+1)(n+2 \alpha+2) P_{n}^{(\alpha, \alpha)}(x) \\
& \left.+[(n-2)(n+2 \alpha+3)+(2 \alpha+3)(2 \alpha+4)]\left(1-x^{2}\right) \frac{d^{2}}{d x^{2}} P_{n}^{(\alpha, \alpha)}(x)\right] \\
& =\frac{4}{(2 \alpha+1)}\left(\begin{array}{c}
n+2 \alpha \\
n
\end{array}\right) \frac{d^{2}}{d x^{2}} P_{n}^{(\alpha, \alpha)}(x) \\
& -\frac{n(n-1)(n+2 \alpha+1)(n+2 \alpha+2)}{(\alpha+1)(\alpha+2)(2 \alpha+1)}\left(\begin{array}{c}
n+2 \alpha \\
n
\end{array}\right) P_{n}^{(\alpha, \alpha)}(x) \\
& =\frac{4}{(2 \alpha+1)}\left(\begin{array}{c}
n+2 \alpha \\
n
\end{array}\right) \frac{d^{2}}{d x^{2}} P_{n}^{(\alpha, \alpha)}(x) \\
& -4(2 \alpha+3)\left(\begin{array}{c}
n+2 \alpha+2 \\
n-2
\end{array}\right) P_{n}^{(\alpha, \alpha)}(x) \text {. }
\end{aligned}
$$

Finally, this implies that

$$
\sum_{i=0}^{\infty} c_{i}(x) D^{i} P_{n}^{(\alpha, \alpha)}(x)=\frac{4}{(2 \alpha+1)}\left(\begin{array}{c}
n+2 \alpha \\
n
\end{array}\right) \frac{d^{2}}{d x^{2}} P_{n}^{(\alpha, \alpha)}(x),
$$

which proves $(25)$. 
The proof of (26) is much easier. We start from

$$
\begin{aligned}
& \sum_{i=2}^{\infty} i c_{i}^{*}(x) D^{i} P_{n}^{(\alpha, \alpha)}(x) \\
& \quad=\sum_{i=0}^{\infty}\left(\begin{array}{c}
\alpha+1 \\
i
\end{array}\right) \frac{2^{i+2}}{(i+1) !} \sum_{k=0}^{\infty} \frac{(-2 \alpha+i-2)_{k}}{k !(i+2)_{k}}(1-x)^{k} D^{i+k+2} P_{n}^{(\alpha, \alpha)}(x) .
\end{aligned}
$$

We use Vandermonde's summation formula (9) again to obtain

$$
\begin{aligned}
& \sum_{k=0}^{\infty} \frac{(-2 \alpha+i-2)_{k}}{k !(i+2)_{k}}(1-x)^{k} D^{i+k+2} P_{n}^{(\alpha, \alpha)}(x) \\
&=\left(\begin{array}{c}
n+\alpha \\
n
\end{array}\right) \sum_{m=0}^{\infty} \sum_{k=0}^{m} \frac{(-2 \alpha+i-2)_{k}}{k !(i+2)_{k}} \\
& \quad \times \frac{(-n)_{m+i+2}(n+2 \alpha+1)_{m+i+2}}{2^{m+i+2}(\alpha+1)_{m+i+2}} \frac{(-m)_{k}}{m !}(-1)^{i}(1-x)^{m} \\
&=\left(\begin{array}{c}
n+\alpha \\
n
\end{array}\right)(-1)^{i} \sum_{m=0}^{\infty} \frac{(-n)_{m+i+2}(n+2 \alpha+1)_{m+i+2}}{2^{m+i+2}(\alpha+1)_{m+i+2}} \frac{(1-x)^{m}}{m !} \\
& \times{ }_{2} F_{1}\left(\begin{array}{c}
-m,-2 \alpha+i-2 \\
i+2
\end{array}\right) \\
&=\left(\begin{array}{c}
n+\alpha \\
n
\end{array}\right)(-1)^{i} \sum_{m=0}^{\infty} \frac{(-n)_{m+i+2}(n+2 \alpha+1)_{m+i+2}}{2^{m+i+2}(\alpha+1)_{m+i+2}} \\
& \times \frac{(1-x)^{m}}{m !} \frac{(2 \alpha+4)_{m}}{(i+2)_{m}} \\
&=\left(\begin{array}{c}
n+\alpha \\
n
\end{array}\right)(-1)^{i} \frac{(i+1) !}{2^{i+2}} \sum_{m=0}^{\infty} \frac{(-n)_{m+i+2}(n+2 \alpha+1)_{m+i+2}}{(m+i+1) !(\alpha+1)_{m+i+2}} \\
& \times \frac{(2 \alpha+4)_{m}}{m !}\left(\frac{1-x}{2}\right)^{m} \cdot
\end{aligned}
$$

This leads to

$$
\begin{aligned}
\sum_{i=2}^{\infty} i c_{i}^{*}(x) D^{i} P_{n}^{(\alpha, \alpha)}(x) \\
=\left(\begin{array}{c}
n+\alpha \\
n
\end{array}\right) \sum_{i=0}^{\infty}(-1)^{i}\left(\begin{array}{c}
\alpha+1 \\
i
\end{array}\right) \\
\quad \times \sum_{m=0}^{\infty} \frac{(-n)_{m+i+2}(n+2 \alpha+1)_{m+i+2}}{(m+i+1) !(\alpha+1)_{m+i+2}} \frac{(2 \alpha+4)_{m}}{m !}\left(\frac{1-x}{2}\right)^{m} \\
=\left(\begin{array}{c}
n+\alpha \\
n
\end{array}\right) \sum_{m=0}^{\infty} \frac{(-n)_{m+2}(n+2 \alpha+1)_{m+2}}{(m+1) !(\alpha+1)_{m+2}} \frac{(2 \alpha+4)_{m}}{m !}\left(\frac{1-x}{2}\right)^{m} \\
\quad \times{ }_{3} F_{2}\left(\begin{array}{c}
-n+m+2,-\alpha-1, n+2 \alpha+m+3 \\
m+2, \alpha+m+3
\end{array}\right) .
\end{aligned}
$$


Now we use the Saalschütz summation formula (10) again to find for $n \geq m+2$

$$
\begin{aligned}
{ }_{3} F_{2}\left(\begin{array}{c}
-n+m+2,-\alpha-1, n+2 \alpha+m+3 \\
m+2, \alpha+m+3
\end{array} \mid 1\right) \\
\quad=\frac{(\alpha+m+3)_{n-m-2}(-n-2 \alpha-1)_{n-m-2}}{(m+2)_{n-m-2}(-n-\alpha)_{n-m-2}} \\
\quad=\frac{\Gamma(n+\alpha+1)}{\Gamma(\alpha+m+3)} \frac{(m+1) !}{\Gamma(n)} \frac{\Gamma(n+2 \alpha+2)}{\Gamma(m+2 \alpha+4)} \frac{\Gamma(\alpha+m+3)}{\Gamma(n+\alpha+1)} \\
\quad=\frac{(m+1) !}{\Gamma(n+2 \alpha+2)} \frac{\Gamma(m+2 \alpha+4)}{\Gamma(m+2)}
\end{aligned}
$$

Since $(-n)_{m+2}=0$ and the ${ }_{3} F_{2}(1)$ exists for $n<m+2$, this implies, by using (4),

$$
\begin{aligned}
\sum_{i=2}^{\infty} i c_{i}^{*}(x) D^{i} P_{n}^{(\alpha, \alpha)}(x) \\
=\left(\begin{array}{c}
n+\alpha \\
n
\end{array}\right) \sum_{m=0}^{\infty} \frac{(-n)_{m+2}(n+2 \alpha+1)_{m+2}}{(m+1) !(\alpha+1)_{m+2}} \frac{(2 \alpha+4)_{m}}{m !} \\
\quad \times \frac{(m+1) !}{\Gamma(n)} \frac{\Gamma(n+2 \alpha+2)}{\Gamma(m+2 \alpha+4)}\left(\frac{1-x}{2}\right)^{m} \\
=\left(\begin{array}{c}
n+\alpha \\
n
\end{array}\right) \frac{\Gamma(n+2 \alpha+2)}{\Gamma(n) \Gamma(2 \alpha+4)} \sum_{m=0}^{\infty} \frac{(-n)_{m+2}(n+2 \alpha+1)_{m+2}}{m !(\alpha+1)_{m+2}}\left(\frac{1-x}{2}\right)^{m} \\
=\frac{4}{(2 \alpha+3)}\left(\begin{array}{c}
n+2 \alpha+1 \\
n-1
\end{array}\right) \frac{d^{2}}{d x^{2}} P_{n}^{(\alpha, \alpha)}(x),
\end{aligned}
$$

and therefore we have

$$
\begin{aligned}
& \sum_{i=2}^{\infty} i c_{i}(x) D^{i} P_{n}^{(\alpha, \alpha)}(x) \\
& \quad=4\left(\begin{array}{c}
n+2 \alpha+1 \\
n-1
\end{array}\right)\left(1-x^{2}\right) \frac{d^{2}}{d x^{2}} P_{n}^{(\alpha, \alpha)}(x) .
\end{aligned}
$$

Now we look at the second sum on the left-hand side of (26). Now we have

$$
\begin{aligned}
& \sum_{i=2}^{\infty} c_{i}^{*}(x) D^{i+1} P_{n}^{(\alpha, \alpha)}(x) \\
& \quad=\sum_{i=0}^{\infty}\left(\begin{array}{c}
\alpha+1 \\
i
\end{array}\right) \frac{2^{i+2}}{(i+2) !} \sum_{k=0}^{\infty} \frac{(-2 \alpha+i-2)_{k}}{k !(i+3)_{k}}(1-x)^{k} D^{i+k+3} P_{n}^{(\alpha, \alpha)}(x)
\end{aligned}
$$


As before we find by using the Vandermonde summation formula (9)

$$
\begin{aligned}
\sum_{k=0}^{\infty} \frac{(-}{-2 \alpha+i-2)_{k}} & k !(i+3)_{k} \\
= & \left(\begin{array}{c}
n+\alpha \\
n
\end{array}\right) \sum_{m=0}^{\infty} \sum_{k=0}^{m} \frac{(-2 \alpha+i-2)_{k}}{k !(i+3)_{k}} \\
& \times \frac{(-n)_{m+i+3}(n+2 \alpha+1)_{m+i+3}}{2^{m+i+3}(\alpha+1)_{m+i+3}} \frac{(-m)_{k}}{m !}(-1)^{i+1}(1-x)^{m} \\
= & \left(\begin{array}{c}
n+\alpha \\
n
\end{array}\right)(-1)^{i+1} \sum_{m=0}^{\infty} \frac{(-n)_{m+i+3}(n+2 \alpha+1)_{m+i+3}}{2^{m+i+3}(\alpha+1)_{m+i+3}} \frac{(1-x)^{m}}{m !} \\
& \times{ }_{2} F_{1}\left(\begin{array}{c}
-m,-2 \alpha+i-\left.2\right|_{1} \\
i+3
\end{array}\right) \\
= & \left(\begin{array}{c}
n+\alpha \\
n
\end{array}\right)(-1)^{i+1} \sum_{m=0}^{\infty} \frac{(-n)_{m+i+3}(n+2 \alpha+1)_{m+i+3}}{2^{m+i+3}(\alpha+1)_{m+i+3}} \frac{(1-x)^{m}}{m !} \frac{(2 \alpha+5)_{m}}{(i+3)_{m}} \\
= & \left(\begin{array}{c}
n+\alpha \\
n
\end{array}\right)(-1)^{i+1} \frac{(i+2) !}{2^{i+3}} \\
& \times \sum_{m=0}^{\infty} \frac{(-n)_{m+i+3}(n+2 \alpha+1)_{m+i+3}}{(m+i+2) !(\alpha+1)_{m+i+3}} \frac{(2 \alpha+5)_{m}}{m !}\left(\frac{1-x}{2}\right)^{m},
\end{aligned}
$$

and therefore we obtain

$$
\begin{aligned}
\sum_{i=2}^{\infty} c_{i}^{*}(x) D^{i+1} P_{n}^{(\alpha, \alpha)}(x) \\
=\frac{1}{2}\left(\begin{array}{c}
n+\alpha \\
n
\end{array}\right) \sum_{i=0}^{\infty}(-1)^{i+1}\left(\begin{array}{c}
\alpha+1 \\
i
\end{array}\right) \\
\quad \times \sum_{m=0}^{\infty} \frac{(-n)_{m+i+3}(n+2 \alpha+1)_{m+i+3}}{(m+i+2) !(\alpha+1)_{m+i+3}} \frac{(2 \alpha+5)_{m}}{m !}\left(\frac{1-x}{2}\right)^{m} \\
=-\frac{1}{2}\left(\begin{array}{c}
n+\alpha \\
n
\end{array}\right) \sum_{m=0}^{\infty} \frac{(-n)_{m+3}(n+2 \alpha+1)_{m+3}}{(m+2) !(\alpha+1)_{m+3}} \frac{(2 \alpha+5)_{m}}{m !}\left(\frac{1-x}{2}\right)^{m} \\
\quad \times{ }_{3} F_{2}\left(\begin{array}{c}
-n+m+3,-\alpha-1, n+2 \alpha+m+4 \\
m+3, \alpha+m+4
\end{array}\right.
\end{aligned}
$$

By using the Saalschütz summation formula (10) we have for $n \geq m+3$

$$
\begin{aligned}
{ }_{3} F_{2}\left(\begin{array}{c}
-n+m+3,-\alpha-1, n+2 \alpha+m+4 \\
m+3, \alpha+m+4
\end{array}\right. \\
\quad=\frac{(\alpha+m+4)_{n-m-3}(-n-2 \alpha-1)_{n-m-3}}{(m+3)_{n-m-3}(-n-\alpha)_{n-m-3}} \\
\quad=\frac{\Gamma(n+\alpha+1)}{\Gamma(\alpha+m+4)} \frac{(m+2) !}{\Gamma(n)} \frac{\Gamma(n+2 \alpha+2)}{\Gamma(m+2 \alpha+5)} \frac{\Gamma(\alpha+m+4)}{\Gamma(n+\alpha+1)} \\
\quad=\frac{(m+2) !}{\Gamma(n)} \frac{\Gamma+2 \alpha+2)}{\Gamma(m+2 \alpha+5)}
\end{aligned}
$$


which gives us by using (4), since $(-n)_{m+3}=0$ and the ${ }_{3} F_{2}(1)$ exists for $n<m+3$ :

$$
\begin{aligned}
\sum_{i=2}^{\infty} c_{i}^{*}(x) D^{i+1} P_{n}^{(\alpha, \alpha)}(x) \\
=-\frac{1}{2}\left(\begin{array}{c}
n+\alpha \\
n
\end{array}\right) \sum_{m=0}^{\infty} \frac{(-n)_{m+3}(n+2 \alpha+1)_{m+3}}{(m+2) !(\alpha+1)_{m+3}} \frac{(2 \alpha+5)_{m}}{m !} \\
\quad \times \frac{(m+2) !}{\Gamma(n)} \frac{\Gamma(n+2 \alpha+2)}{\Gamma(m+2 \alpha+5)}\left(\frac{1-x}{2}\right)^{m} \\
=-\frac{1}{2}\left(\begin{array}{c}
n+\alpha \\
n
\end{array}\right) \frac{\Gamma(n+2 \alpha+2)}{\Gamma(n) \Gamma(2 \alpha+5)} \sum_{m=0}^{\infty} \frac{(-n)_{m+3}(n+2 \alpha+1)_{m+3}}{m !(\alpha+1)_{m+3}}\left(\frac{1-x}{2}\right)^{m} \\
=\frac{d^{3}}{(2 \alpha+3)(2 \alpha+4)}\left(\begin{array}{c}
n+2 \alpha+1 \\
n-1
\end{array}\right) \frac{d_{n}^{(\alpha, \alpha)}(x) .}{d x^{3}} P_{n}^{m}
\end{aligned}
$$

This implies

$$
\begin{gathered}
\sum_{i=2}^{\infty} c_{i}(x) D^{i+1} P_{n}^{(\alpha, \alpha)}(x)=\frac{4}{(2 \alpha+4)}\left(\begin{array}{c}
n+2 \alpha+1 \\
n-1
\end{array}\right)\left(1-x^{2}\right) \frac{d^{3}}{d x^{3}} P_{n}^{(\alpha, \alpha)}(x) \\
=\frac{4}{(2 \alpha+4)}\left(\begin{array}{c}
n+2 \alpha+1 \\
n-1
\end{array}\right)\left[\begin{array}{c}
2(\alpha+2) x \frac{d^{2}}{d x^{2}} P_{n}^{(\alpha, \alpha)}(x) \\
\left.-(n-1)(n+2 \alpha+2) \frac{d}{d x} P_{n}^{(\alpha, \alpha)}(x)\right]
\end{array}\right.
\end{gathered}
$$

Now we have found

$$
\begin{aligned}
\sum_{i=0}^{\infty} i c_{i}(x) D^{i} P_{n}^{(\alpha, \alpha)}(x)+x \sum_{i=0}^{\infty} c_{i}(x) D^{i+1} P_{n}^{(\alpha, \alpha)}(x) \\
=4\left(\begin{array}{c}
n+2 \alpha+1 \\
n-1
\end{array}\right)\left(1-x^{2}\right) \frac{d^{2}}{d x^{2}} P_{n}^{(\alpha, \alpha)}(x) \\
+4(2 \alpha+3)\left(\begin{array}{c}
n+2 \alpha+2 \\
n-2
\end{array}\right) x \frac{d}{d x} P_{n}^{(\alpha, \alpha)}(x) \\
+\frac{4 x}{(2 \alpha+4)}\left(\begin{array}{c}
n+2 \alpha+1 \\
n-1
\end{array}\right) \\
\quad \times\left[\begin{array}{c}
\left.2(\alpha+2) x \frac{d^{2}}{d x^{2}} P_{n}^{(\alpha, \alpha)}(x)-(n-1)(n+2 \alpha+2) \frac{d}{d x} P_{n}^{(\alpha, \alpha)}(x)\right] \\
=
\end{array}\right. \\
\quad\left(\begin{array}{c}
n+2 \alpha+1 \\
n-1
\end{array}\right) \frac{d^{2}}{d x^{2}} P_{n}^{(\alpha, \alpha)}(x)
\end{aligned}
$$

which proves (26) and therefore Theorem 2.

\section{DifFERENTIAL EQUATIONS FOR $P_{n}^{\alpha, \pm 1 / 2,0, N}(x)$}

In this section we will derive a differential equation for the polynomials $\left\{P_{n}^{\alpha,-1 / 2,0, N}(x)\right\}_{n=0}^{\infty}$ and another one for the polynomials $\left\{P_{n}^{\alpha, 1 / 2,0, N}(x)\right\}_{n=0}^{\infty}$ for all $\alpha>-1$ and $N \geq 0$. These differential equations can be obtained from 
our results by applying the following quadratic transformations (see [7]):

$$
\frac{P_{2 n}^{\alpha, \alpha, M, M}(x)}{P_{2 n}^{\alpha, \alpha, M, M}(1)}=\frac{P_{n}^{\alpha,-1 / 2,0,2 M}\left(2 x^{2}-1\right)}{P_{n}^{\alpha,-1 / 2,0,2 M}(1)}
$$

and

$$
\frac{P_{2 n+1}^{\alpha, \alpha, M, M}(x)}{P_{2 n+1}^{\alpha, \alpha, M, M}(1)}=\frac{x P_{n}^{\alpha, 1 / 2,0,(4 \alpha+6) M}\left(2 x^{2}-1\right)}{P_{n}^{\alpha, 1 / 2,0,(4 \alpha+6) M}(1)} .
$$

Note that (27) and (28) reduce to (7) and (8) if $M=0$.

If we set $y(x):=f\left(2 x^{2}-1\right)$, we can prove by induction that

$$
y^{(2 i)}(x)=\sum_{j=i}^{2 i} \frac{(2 i) ! 2^{3 j-2 i}}{(2 j-2 i) !(2 i-j) !} x^{2 j-2 i} f^{(j)}\left(2 x^{2}-1\right), \quad i=0,1,2, \ldots,
$$

and

$$
\begin{array}{r}
y^{(2 i+1)}(x)=\sum_{j=i+1}^{2 i+1} \frac{(2 i+1) ! 2^{3 j-2 i-1}}{(2 j-2 i-1) !(2 i-j+1) !} x^{2 j-2 i-1} f^{(j)}\left(2 x^{2}-1\right), \\
\quad i=0,1,2, \ldots,
\end{array}
$$

or written in one formula

$$
\begin{array}{rl}
y^{(i)}(x)=\sum_{j=[(i+1) / 2]}^{i} \frac{i ! 2^{3 j-i}}{(2 j-i) !(i-j) !} x^{2 j-i} f^{(j)}\left(2 x^{2}-1\right) & \\
i & i=0,1,2, \ldots .
\end{array}
$$

Note that (27) and (29) substituted in the differential equation given in Theorem 1 leads to a triviality, since

$$
\begin{aligned}
\sum_{i=1}^{\infty} b_{i}(x) y^{(i)}(x) & =\sum_{i=1}^{\infty} \sum_{j=[(i+1) / 2]}^{i} \frac{(-1)^{i} 2^{3 j-1}}{(2 j-i) !(i-j) !} x^{2 j} f^{(j)}\left(2 x^{2}-1\right) \\
& =\sum_{j=1}^{\infty} 2^{3 j-1} x^{2 j} f^{(j)}\left(2 x^{2}-1\right) \sum_{i=j}^{2 j} \frac{(-1)^{i}}{(2 j-i) !(i-j) !}=0,
\end{aligned}
$$

in view of

$$
\sum_{i=j}^{2 j} \frac{(-1)^{i}}{(2 j-i) !(i-j) !}=\frac{(-1)^{j}}{j !} \sum_{i=0}^{j}\left(\begin{array}{l}
j \\
i
\end{array}\right)(-1)^{i}=0, \quad j=1,2,3, \ldots
$$

By using (27) and (29) we obtain from Theorem 2 the following equation:

$$
\begin{aligned}
& M \sum_{i=0}^{\infty} c_{i}(x) \sum_{j=[(i+1) / 2]}^{i} \frac{i ! 2^{3 j-i}}{(2 j-i) !(i-j) !} x^{2 j-i} f^{(j)}\left(2 x^{2}-1\right) \\
& \quad+\left(1-x^{2}\right)\left[16 x^{2} f^{\prime \prime}\left(2 x^{2}-1\right)+4 f^{\prime}\left(2 x^{2}-1\right)\right] \\
& \quad-8(\alpha+1) x^{2} f^{\prime}\left(2 x^{2}-1\right)+2 n(2 n+2 \alpha+1) f\left(2 x^{2}-1\right)=0
\end{aligned}
$$

with

$$
c_{0}(x):=c_{0}(2 n, \alpha, x)=4(2 \alpha+3)\left(\begin{array}{c}
2 n+2 \alpha+2 \\
2 n-2
\end{array}\right), \quad n=0,1,2, \ldots,
$$


satisfied by

$$
f(x):=P_{n}^{\alpha,-1 / 2,0,2 M}(x)
$$

Since

$$
\begin{aligned}
& \sum_{i=1}^{\infty} c_{i}(x) \sum_{j=[(i+1) / 2]}^{i} \frac{i ! 2^{3 j-i}}{(2 j-i) !(i-j) !} x^{2 j-i} f^{(j)}\left(2 x^{2}-1\right) \\
& =\sum_{j=1}^{\infty} 2^{3 j} f^{(j)}\left(2 x^{2}-1\right) \sum_{i=j}^{2 j} \frac{i ! 2^{-i}}{(2 j-i) !(i-j) !} x^{2 j-i} c_{i}(x),
\end{aligned}
$$

we obtain

$$
\begin{aligned}
& M \sum_{j=0}^{\infty} d_{j}(x) f^{(j)}\left(2 x^{2}-1\right)+\left(1-x^{2}\right)\left[16 x^{2} f^{\prime \prime}\left(2 x^{2}-1\right)+4 f^{\prime}\left(2 x^{2}-1\right)\right] \\
& \quad-8(\alpha+1) x^{2} f^{\prime}\left(2 x^{2}-1\right)+2 n(2 n+2 \alpha+1) f\left(2 x^{2}-1\right)=0
\end{aligned}
$$

where

$$
\left\{\begin{array}{l}
d_{0}(x)=4(2 \alpha+3)\left(\begin{array}{c}
2 n+2 \alpha+2 \\
2 n-2
\end{array}\right), \quad n=0,1,2, \ldots, \\
d_{j}(x)=\sum_{i=j}^{2 j} \frac{i ! 2^{3 j-i}}{(2 j-i) !(i-j) !} x^{2 j-i} c_{i}(x), \quad j=1,2,3, \ldots
\end{array}\right.
$$

By using (19) and (23) we easily see that $d_{j}(x)$ is an even polynomial with degree $\left[d_{j}(x)\right] \leq 2 j$ for each $j=1,2,3, \ldots$. Now we set $2 x^{2}-1=t$ and $N=2 M$ in (30) to find a differential equation of the form

$$
\begin{aligned}
& N \sum_{j=0}^{\infty} d_{j}^{*}(t) y^{(j)}(t)+\left(1-t^{2}\right) y^{\prime \prime}(t) \\
& \quad-\frac{1}{2}[(2 \alpha+1)+(2 \alpha+3) t] y^{\prime}(t)+\frac{1}{2} n(2 n+2 \alpha+1) y(t)=0,
\end{aligned}
$$

for the polynomials $\left\{P_{n}^{\alpha,-1 / 2,0, N}(t)\right\}_{n=0}^{\infty}$, where

$$
d_{0}^{*}(t)=\frac{1}{2}(2 \alpha+3)\left(\begin{array}{c}
2 n+2 \alpha+2 \\
2 n-2
\end{array}\right), \quad n=0,1,2, \ldots,
$$

and

$$
d_{j}^{*}(t)=\frac{1}{8} d_{j}\left(\sqrt{\frac{1+t}{2}}\right), \quad j=1,2,3, \ldots
$$

We remark that $d_{j}^{*}(t)$ is a polynomial in $t$ with degree $\left[d_{j}^{*}(t)\right] \leq j$ for every $j=1,2,3, \ldots$. By using (19) and (23) we see that

$$
\begin{aligned}
d_{j}^{*}(-1) & =\frac{1}{8} d_{j}(0)=\frac{(2 j) ! 2^{j-3}}{j !} c_{2 j}(0) \\
& =(2 \alpha+3)(-1)^{j+1}\left(\begin{array}{c}
\alpha+1 \\
j-1
\end{array}\right) \frac{2^{j-1}}{j !} \neq 0, \quad j=1,2,3, \ldots,
\end{aligned}
$$


if $\alpha$ is not a nonnegative integer. This implies that the order of the differential equation is infinite in that case if $N>0$. For nonnegative integer values of $\alpha$ we have $d_{j}(x)=0$ for $j>2 \alpha+4$ and

$$
d_{2 \alpha+4}(x)=2^{4 \alpha+8} x^{2 \alpha+4} c_{2 \alpha+4}(x) \neq 0
$$

since degree $\left[c_{2 \alpha+4}(x)\right]=2 \alpha+4$. This implies that the order of this differential equation equals $2 \alpha+4$ if $\alpha$ is a nonnegative integer and $N>0$.

And if we set $y(x):=x f\left(2 x^{2}-1\right)$, we find by using Leibniz's rule

$$
\begin{aligned}
y^{(2 i)}(x)= & x \sum_{j=i}^{2 i} \frac{(2 i) ! 2^{3 j-2 i}}{(2 j-2 i) !(2 i-j) !} x^{2 j-2 i} f^{(j)}\left(2 x^{2}-1\right) \\
& +2 i \sum_{j=i}^{2 i-1} \frac{(2 i-1) ! 2^{3 j-2 i+1}}{(2 j-2 i+1) !(2 i-1-j) !} x^{2 j-2 i+1} f^{(j)}\left(2 x^{2}-1\right) \\
= & \sum_{j=i}^{2 i} \frac{(2 i+1) ! 2^{3 j-2 i}}{(2 j-2 i+1) !(2 i-j) !} x^{2 j-2 i+1} f^{(j)}\left(2 x^{2}-1\right), \quad i=0,1,2, \ldots,
\end{aligned}
$$

and

$$
\begin{aligned}
y^{(2 i+1)}(x)= & x \sum_{j=i+1}^{2 i+1} \frac{(2 i+1) ! 2^{3 j-2 i-1}}{(2 j-2 i-1) !(2 i-j+1) !} x^{2 j-2 i-1} f^{(j)}\left(2 x^{2}-1\right) \\
& +(2 i+1) \sum_{j=i}^{2 i} \frac{(2 i) ! 2^{3 j-2 i}}{(2 j-2 i) !(2 i-j) !} x^{2 j-2 i} f^{(j)}\left(2 x^{2}-1\right) \\
= & \sum_{j=i}^{2 i+1} \frac{(2 i+2) ! 2^{3 j-2 i-1}}{(2 j-2 i) !(2 i-j+1) !} x^{2 j-2 i} f^{(j)}\left(2 x^{2}-1\right), \quad i=0,1,2, \ldots,
\end{aligned}
$$

or written in one formula

$$
\begin{array}{r}
y^{(i)}(x)=\sum_{j=[i / 2]}^{i} \frac{(i+1) ! 2^{3 j-i}}{(2 j-i+1) !(i-j) !} x^{2 j-i+1} f^{(j)}\left(2 x^{2}-1\right), \\
i=0,1,2, \ldots
\end{array}
$$

If we substitute (28) and (31) in the differential equation in Theorem 1, we find a triviality, since

$$
\begin{aligned}
y(x) & +\sum_{i=1}^{\infty} b_{i}(x) y^{(i)}(x) \\
& =x f\left(2 x^{2}-1\right)+\sum_{i=1}^{\infty} \sum_{j=[i / 2]}^{i} \frac{(i+1)(-1)^{i} 2^{3 j-1}}{(2 j-i+1) !(i-j) !} x^{2 j+1} f^{(j)}\left(2 x^{2}-1\right) \\
& =\sum_{j=1}^{\infty} 2^{3 j-1} x^{2 j+1} f^{(j)}\left(2 x^{2}-1\right) \sum_{i=j}^{2 j+1} \frac{(i+1)(-1)^{i}}{(2 j-i+1) !(i-j) !}=0
\end{aligned}
$$


in view of

$$
\begin{aligned}
\sum_{i=j}^{2 j+1} \frac{(i+1)(-1)^{i}}{(2 j-i+1) !(i-j) !} & =\sum_{i=0}^{j+1} \frac{(i+j+1)(-1)^{i+j}}{(j-i+1) ! i !} \\
& =\frac{(-1)^{j}}{j !}\left[\begin{array}{c}
j+1 \\
i=0
\end{array}\left(\begin{array}{c}
j+1 \\
i
\end{array}\right)(-1)^{i}-\sum_{i=0}^{j}\left(\begin{array}{l}
j \\
i
\end{array}\right)(-1)^{i}\right] \\
& =0, \quad j=1,2,3, \ldots
\end{aligned}
$$

By using (28) and (31) we obtain from Theorem 2 the following equation:

$$
\begin{aligned}
M \sum_{i=0}^{\infty} c_{i}(x) \sum_{j=[i / 2]}^{i} \frac{(i+1) ! 2^{3 j-i}}{(2 j-i+1) !(i-j) !} x^{2 j-i+1} f^{(j)}\left(2 x^{2}-1\right) \\
\quad+\left(1-x^{2}\right)\left[16 x^{3} f^{\prime \prime}\left(2 x^{2}-1\right)+12 x f^{\prime}\left(2 x^{2}-1\right)\right] \\
\quad-2(\alpha+1) x\left[4 x^{2} f^{\prime}\left(2 x^{2}-1\right)+f\left(2 x^{2}-1\right)\right] \\
\quad+2(2 n+1)(n+\alpha+1) x f\left(2 x^{2}-1\right)=0
\end{aligned}
$$

with

$$
c_{0}(x):=c_{0}(2 n+1, \alpha, x)=4(2 \alpha+3)\left(\begin{array}{c}
2 n+2 \alpha+3 \\
2 n-1
\end{array}\right), \quad n=0,1,2, \ldots,
$$

satisfied by

Since

$$
f(x):=P_{n}^{\alpha, 1 / 2,0,(4 \alpha+6) M}(x) .
$$

$$
\begin{aligned}
& \sum_{i=0}^{\infty} c_{i}(x) \sum_{j=[i / 2]}^{i} \frac{(i+1) ! 2^{3 j-i}}{(2 j-i+1) !(i-j) !} x^{2 j-i+1} f^{(j)}\left(2 x^{2}-1\right) \\
& =\sum_{j=0}^{\infty} 2^{3 j} f^{(j)}\left(2 x^{2}-1\right) \sum_{i=j}^{2 j+1} \frac{(i+1) ! 2^{-i}}{(2 j-i+1) !(i-j) !} x^{2 j-i+1} c_{i}(x)
\end{aligned}
$$

we obtain, after division by $x$,

$$
\begin{aligned}
& M \sum_{j=0}^{\infty} e_{j}(x) f^{(j)}\left(2 x^{2}-1\right)+\left(1-x^{2}\right)\left[16 x^{2} f^{\prime \prime}\left(2 x^{2}-1\right)+12 f^{\prime}\left(2 x^{2}-1\right)\right] \\
& \quad-2(\alpha+1)\left[4 x^{2} f^{\prime}\left(2 x^{2}-1\right)+f\left(2 x^{2}-1\right)\right] \\
& \quad+2(2 n+1)(n+\alpha+1) f\left(2 x^{2}-1\right)=0
\end{aligned}
$$

where

$$
e_{j}(x)=\sum_{i=j}^{2 j+1} \frac{(i+1) ! 2^{3 j-i}}{(2 j-i+1) !(i-j) !} x^{2 j-i} c_{i}(x), \quad j=0,1,2, \ldots
$$

By using (19) and (23) we easily see that $e_{j}(x)$ is an even polynomial with degree $\left[e_{j}(x)\right] \leq 2 j$ for each $j=0,1,2, \ldots$. Now we set $2 x^{2}-1=t$ and $N=(4 \alpha+6) M$ in (32) to find a differential equation of the form

$$
\begin{aligned}
& N \sum_{j=0}^{\infty} e_{j}^{*}(t) y^{(j)}(t)+\left(1-t^{2}\right) y^{\prime \prime}(t) \\
& \quad-\frac{1}{2}[(2 \alpha-1)+(2 \alpha+5) t] y^{\prime}(t)+\frac{1}{2} n(2 n+2 \alpha+3) y(t)=0,
\end{aligned}
$$


for the polynomials $\left\{P_{n}^{\alpha, 1 / 2,0, N}(t)\right\}_{n=0}^{\infty}$, where

$$
e_{0}^{*}(t)=\frac{1}{2}\left(\begin{array}{c}
2 n+2 \alpha+3 \\
2 n-1
\end{array}\right), \quad n=0,1,2, \ldots
$$

and

$$
e_{j}^{*}(t)=\frac{1}{8(2 \alpha+3)} e_{j}\left(\sqrt{\frac{1+t}{2}}\right), \quad j=1,2,3, \ldots .
$$

Note that $e_{j}^{*}(t)$ is a polynomial in $t$ with degree $\left[e_{j}^{*}(t)\right] \leq j$ for every $j=$ $1,2,3, \ldots$. By using (19) and (23) we see that

$$
\begin{aligned}
e_{j}^{*}(-1) & =\frac{1}{8(2 \alpha+3)} e_{j}(0)=(-1)^{j+1} \frac{2^{j-1}}{j !}\left[2\left(\begin{array}{c}
\alpha \\
j-1
\end{array}\right)(\alpha+1)+(2 j+1)\left(\begin{array}{c}
\alpha+1 \\
j-1
\end{array}\right)\right] \\
& =(2 \alpha+5)(-1)^{j+1}\left(\begin{array}{c}
\alpha+1 \\
j-1
\end{array}\right) \frac{2^{j-1}}{j !} \neq 0, \quad j=1,2,3, \ldots,
\end{aligned}
$$

if $\alpha$ is not a nonnegative integer. This implies that the order of the differential equation is infinite in that case if $N>0$. For nonnegative integer values of $\alpha$ we have $e_{j}(x)=0$ for $j>2 \alpha+4$ and

$$
e_{2 \alpha+4}(x)=2^{4 \alpha+8} x^{2 \alpha+4} c_{2 \alpha+4}(x) \neq 0 .
$$

This implies that the order of this differential equation equals $2 \alpha+4$ for nonnegative integer values of $\alpha$ and $N>0$.

\section{ACKNOWLEDGMENTS}

I wish to thank Francisco Marcellan and his colleagues for their invitation and hospitality during my visit to Madrid in June 1991. During this stay in Madrid the proof of Theorem 2 was completed.

Many thanks are due to my uncle Jan Koekoek for carefully reviewing earlier versions of this manuscript and for his useful ideas and advice concerning the last section of this paper.

\section{REFERENCES}

1. T. J. I'A. Bromwich, An introduction to the theory of infinite series, 2nd ed., Macmillan, New York, 1959.

2. T. S. Chihara, An introduction to orthogonal polynomials, Math. and Its Appl., vol. 13, Gordon and Breach, New York, 1978.

3. A. Erdélyi et al. (Eds.), Higher transcendental functions, Bateman Manuscript Project, Vol. I, McGraw-Hill, New York, 1953.

4. W. N. Everitt and L. L. Littlejohn, Orthogonal polynomials and spectral theory: a survey, Orthogonal Polynomials and their Applications (C. Brezinski, L. Gori, and A. Ronveaux, eds.), IMACS Annals on Computing and Applied Mathematics, vol. 9, J. C. Baltzer A. G., 1991, pp. 21-55.

5. J. Koekoek and R. Koekoek, On a differential equation for Koornwinder's generalized Laguerre polynomials, Proc. Amer. Math. Soc. 112 (1991), 1045-1054.

6. R. Koekoek, The search for differential equations for certain sets of orthogonal polynomials, J. Comput. Appl. Math. 49 (1993), 111-119.

7. T. H. Koornwinder, Orthogonal polynomials with weight function $(1-x)^{\alpha}(1+x)^{\beta}+M \delta(x+1)$ $+N \delta(x-1)$, Canad. Math. Bull. (2) 27 (1984), 205-214. 
8. A. M. Krall, Orthogonal polynomials satisfying fourth order differential equations, Proc. Royal Soc. Edinburgh Sect. A 87 (1981), 271-288.

9. A. M. Krall and L. L. Littlejohn, On the classification of differential equations having orthogonal polynomial solutions. II, Ann. Mat. Pura Appl. (4) 149 (1987), 77-102.

10. H. L. Krall, Certain differential equations for Tchebycheff polynomials, Duke Math. J. 4 (1938), 705-718.

11. $\ldots$ On orthogonal polynomials satisfying a certain fourth order differential equation, The Pennsylvania State College Studies, No. 6, 1940.

12. L. L. Littlejohn, The Krall polynomials: A new class of orthogonal polynomials, Quaestiones Math. 5 (1982), 255-265.

13. _ The Krall polynomials as solutions to a second order differential equation, Canad. Math. Bull. 26 (1983), 410-417.

14. __ On the classification of differential equations having orthogonal polynomial solutions, Ann. Mat. Pura Appl. (4) 93 (1984), 35-53.

15. L. L. Litlejohn and S. D. Shore, Nonclassical orthogonal polynomials as solutions to second order differential equations, Canad. Math. Bull. 25 (1982), 291-295.

16. G. Szegö, Orthogonal polynomials, Amer. Math. Soc. Colloq. Publ., vol. 23, Amer. Math. Soc., Providence, RI, 1939; 4th ed., 1975.

Delft University of Technology, Faculty of Technical Mathematics and Informatics, Mekelweg 4, 2628 CD Delft, The Netherlands

E-mail address: koekoek@twi.tudelft.nl 